\title{
La partition des nutriments entre fonctions physiologiques chez les vaches laitières dépend du génotype et de son expression dans le temps
}

\author{
N.C. FRIGGENS ${ }^{1,2}$, O. MARTIN ${ }^{1,2}$, L. BRUN-LAFLEUR ${ }^{3,4,5}$, D. SAUVANT $T^{1,2}$, P. FAVERDIN $N^{3,4}$ \\ ${ }^{1}$ INRA, UMR0791 MoSAR, 16 rue Claude Bernard, F-75231 Paris, France \\ 2 AgroParisTech, UMR0791 MoSAR, 16 rue Claude Bernard, F-75231 Paris, France \\ 3 INRA, UMR1348 PEGASE, F-35590 Saint-Gilles, France \\ 4 Agrocampus-Ouest, UMR1348 PEGASE, F-35000 Rennes, France \\ ${ }^{5}$ Institut de l'Élevage, F-35652 Le Rheu, France \\ Courriel : nicolas.friggens@agroparistech.fr
}

Les enjeux de durabilité de l'élevage incitent à mettre en œuvre une approche plus globale de la conduite alimentaire des vaches laitières. La partition des nutriments vers les différentes fonctions est au cœur de l'efficience globale du troupeau. Comprendre et prévoir les effets combinés de la génétique et de la conduite d'élevage sur cette partition constitue un défi pour la recherche et un enjeu pour les futurs outils de conseil en élevage ${ }^{1}$.

Les nutriments issus des aliments ingérés par l'animal sont répartis dans des proportions variables vers les différentes fonctions physiologiques (la lactation, la gestion des réserves, la reproduction...). La compréhension des facteurs qui sous-tendent la partition de l'énergie a longtemps été vue comme le moyen de contrôler et maximiser les seules fonctions productives. Pour savoir si un changement d'apports alimentaires va produire, ou non, un bénéfice économique en termes de quantité ou de qualité du lait produit, il est nécessaire de pouvoir prévoir au mieux la partition des nutriments (voir encadré « définitions »). Cette approche de la partition des nutriments se concentre uniquement sur la valeur économique directe, souvent à court terme, avec peu de considération des aspects plus larges de la partition des nutriments ainsi que du plus long terme. Il est cependant clair aujourd'hui que la compréhension de la partition des nutriments est essentielle pour une gamme beaucoup plus large de questions qui sont d'une importance majeure pour le futur de l'élevage.

La proportion des nutriments qui ne sont pas orientés vers les réserves corporelles ou le lait peut également impacter l'environnement (devenir des productions de $\mathrm{C}, \mathrm{N}$ et $\mathrm{P}$ ) non seulement par $\mathrm{kg}$ de lait produit, mais aussi en termes d'efficacité sur l'ensemble de la vie productive des vaches laitières. Ainsi, si on considère l'impact environnemental sur l'ensemble de la carrière de la vache, plus la vie productive est longue et plus la phase d'élevage est courte en proportion, plus grande est l'efficacité. Dans ce contexte, la part des nutriments qui va vers des fonctions telles que la capacité immunitaire et la reproduction peut être d'une importance majeure. Ces fonctions sont également importantes pour le bien-être de l'animal, mais aussi pour la gestion du troupeau et donc la charge de travail.

Les demandes de la société pour une agriculture plus durable sont susceptibles d'accroître la diversité des environnements dans lesquels les élevages seront placés. De plus, les évolutions du climat et l'accroissement de la demande sur les usages des terres et des ressources agricoles, par exemple la demande énergétique, pourraient exacerber cette situation. Il y a un donc un besoin croissant de pouvoir prévoir avec précision la capacité d'un animal à répondre aux défis nutritionnels qui se posent en fonction de l'environnement dans lequel il est placé. Différentes questions peuvent être déclinées à partir de ces considérations. Ainsi, comment la régulation de la partition des nutriments modifie-t-elle la capacité de l'animal à s'adapter, c'est-àdire sa robustesse, lorsque les ressources sont limitées ou déséquilibrées? Ou bien, est-ce que la sélection génétique orientée vers une production intensive est bien adaptée à des systèmes économes à bas intrants ? Ou encore, comment la sélection a-t-elle fait évoluer la capacité d'adaptation des animaux d'élevage?

Ces questions de variations des partitions face à celles des ressources sont importantes, mais le rôle des déterminants animaux spécifiques dans la partition des nutriments pose aussi question. Il n'est pas possible de ne répondre à ces questions qu'avec des modèles de partition des nutriments qui ne concernent que la vache moyenne, considérée en milieu de lactation et produisant une quantité standard de lait. Il est devenu de plus en plus clair qu'il faut être capable de prédire la dynamique de la partition des nutriments d'une manière plus globale et détaillée à la fois, c'està-dire en prenant en compte l'ensemble des facteurs du milieu, des stades physiologiques et des génotypes.

\footnotetext{
${ }^{1}$ Ce texte est adapté d'une publication en langue anglaise publiée dans la revue Animal (Friggens et al 2013).
} 


\section{Encadré. Définitions.}

\section{Partition des nutriments}

Partage des substances nutritives disponibles dans l'organisme entre les différentes voies d'approvisionnement des organes.

\section{Homéostase}

Propriété de régulation associée au concept de « constance du milieu intérieur » (Bernard $1865)$; aptitude d'un système vivant à maintenir un état d'équilibre par le jeu de mécanismes physiologiques contribuant à rétablir cet état en réponse à une perturbation externe (Cannon 1929).

\section{Trajectoires génétiquement déterminées}

Changements d'état au cours du temps dont la force motrice est le « programme » dynamique inné d'expression des gènes de l'animal.

\section{Homéorhèse}

Extension de la notion d'homéostase pour exprimer l'idée de constance des trajectoires génétiquement déterminées (Waddington 1957) ; aptitude d'un système vivant à se maintenir sur une trajectoire d'équilibre par le jeu de mécanismes physiologiques contribuant à rétablir cette trajectoire en réponse à une perturbation externe.

\section{Téléonomie}

Concept scientifique qui reconnaît objectivement le caractère finalisé d'un processus sans pour autant supposer que cette fin soit projetée (Pittendrigh 1958); qualité apparente des systèmes vivants à organiser leur structure et à orchestrer leur fonctionnement pour la réalisation de buts.

Ces nouveaux objectifs exigeront une reconnaissance explicite du rôle du génotype et de son expression à travers le temps sur la partition des nutriments. Dans ce domaine, il y a eu des avancées significatives dans la description de ces trajectoires dont la force motrice est liée à un déterminisme génétique (voir encadré), avec l'émergence de deux approches distinctes. L'une privilégie l'approche ascendante, ou « bottom-up », essayant de modéliser les trajectoires comme une propriété émergente d'un système complexe. L'autre, à l'opposé, est descendante, ou «top-down »; elle met en avant d'emblée ces trajectoires comme des finalités du système qui vont modifier le comportement des systèmes complexes sous-jacents.

Cet article de synthèse analysera tout d'abord ces deux approches dans leur capacité à prendre en compte la partition des nutriments à travers les différents modèles de la lactation qui ont été publiés sur ces aspects. Ensuite, il s'attachera à analyser le défi que représente l'intégration, dans ces modèles, des informations génétiques sur la partition des nutriments. Enfin, la dernière partie tentera de faire le point sur la capacité d'adaptation, ou capacité homéostatique, de l'animal pour faire face à des perturbations environnementales et son lien avec le génotype.

Cette revue privilégie les aspects de prévision et donc inévitablement les approches de modélisation. Cependant, les concepts biologiques impliqués sont également pertinents dans le contexte plus large de la nutrition et de la physio- logie (Friggens et Newbold 2007), ce que le texte essayera de refléter. Cette synthèse se focalise principalement sur les systèmes de contrôle des réseaux métaboliques, ce qui ne veut pas dire qu'il n'y a pas eu des progrès dans la représentation de la "machinerie métabolique » elle-même, mais il n'est pas possible de couvrir tous les aspects de la partition des nutriments dans un seul article de synthèse. Pour de plus amples informations sur les progrès récents sur cet aspect important des modèles de partition des éléments nutritifs, le lecteur pourra, par exemple, consulter les articles de Hanigan et al (2006), Kebreab et al (2009) et Sauvant et al (2010).

\section{1 / Deux approches concep- tuelles en apparence oppo- sées pour prévoir les tra- jectoires de partition des nutriments}

\section{1 / L'évolution de la modélisa- tion des trajectoires génétique- ment déterminées}

La partition des nutriments évolue au cours de la vie de l'animal et de son cycle de reproduction, en particulier pendant les phases de lactation et de gestation. Les vaches en début de lactation orientent une grande partie des nutriments ingérés vers la glande mammaire et mobilisent largement les réserves corporelles disponibles (Bauman et Currie 1980). Au fur et à mesure de l'avancée en lactation des vaches, et en relation avec la progression de la gestation, la partition de l'énergie se déplace de la fonction lactée vers les réserves corporelles (Koenen et al 2001, Yan et al 2006). Les changements qui en résultent dans les réserves corporelles sont similaires pour les différentes races et les cycles de reproduction, et interviennent même lorsque la densité énergétique de la ration est adéquate ou que sa composition est maintenue constante pendant toute la lactation (Friggens et Badsberg 2007). Des évolutions similaires des profils des réserves corporelles sont également observées dans d'autres espèces d'herbivores (Réale et Festa-Bianchet 2000), par exemple pour s'adapter à la période de la demande d'énergie maxi male en fin de gestation chez les brebis qui portent plusieurs agneaux (Kaske et Groth 1997).

Il existe également des profils caractéristiques des courbes de production de matières grasses, de protéines et de lactose du lait qui ne peuvent être attribués à des changements dans la disponibilité ou la composition de la ration offerte (Friggens et al 2007). Les généticiens qui évaluent les particularités phénotypiques propres aux animaux après corrections des effets de l'environnement ont depuis longtemps décrit ces évolutions. Les changements des proportions relatives de ces composants dans le lait au cours de la lactation fournissent une preuve supplémentaire de changements programmés dans la partition des nutriments. Bien entendu, ces changements inscrits dans le génome peuvent traduire une adaptation évolutive. Ainsi, la sécrétion du lactose n'est pas maximale à la parturition, elle s'accroît « génétiquement " en suivant la trajectoire " génétique » d'ingestion de la matière sèche qui constitue la source indirecte majeure de la néoglucogénèse hépatique, l'animal n'ayant pas de réserves de glucose mobilisable.

La raison longtemps évoquée pour expliquer la mobilisation énergétique des réserves corporelles en début de lactation (bilan énergétique négatif) a été l'incapacité à ingérer suffisamment d'énergie pour répondre aux besoins énergétiques liés à la production laitière. Cependant, l'augmentation du contenu énergétique des rations en début de lactation ne permet pas de réduire la mobilisation corporelle (van Knegsel et al 2005, Faverdin et al 2007, Roche et al 2009), l'essentiel du supplément de nutriments apportés étant alors orienté vers la mamelle. Si l'apport en début de lactation était limité parce que les vaches ne peuvent pas manger suffisamment pour satisfaire leurs besoins, la consommation de vaches en bilan énergétique négatif devrait être positivement liée au 
poids vif et la motivation à consommer en début de repas devrait être maximale, ce qui n'est pas le cas (Faverdin 1985, Friggens et Badsberg 2007). De même, l'apport d'aliments concentrés moins encombrants devrait entraîner de faibles phénomènes de substitution avec les fourrages lorsque les vaches sont en déficit, ce qui n'est pas ce que l'on observe expérimentalement en début de lactation (Faverdin et al 2007). Ces résultats, et de nombreux autres, prouvent bien qu'une partie importante de la mobilisation de l'énergie corporelle des vaches laitières en début de lactation est contrôlée génétiquement. Cela implique que la faible ingestion en début de lactation est une conséquence de la partition des nutriments des réserves vers le lait et non la cause de cette partition. En effet, les changements orchestrés dans les principales hormones métaboliques en début de lactation favorisent la lipolyse (Chilliard et al 2000) et créent une véritable « autoroute métabolique » d'orientation des nutriments vers la glande mammaire (Lucy 2003). En outre, ce changement hormonal axé sur la lipolyse tend à réduire l'ingestion d'aliment (Bareille et al 1997, Faverdin et Bareille 1999).

La nécessité de représenter ces différentes trajectoires dans les modèles afin de prévoir la partition des éléments nutritifs est admise depuis longtemps (Baldwin et al 1987, Oldham et Emmans 1989, Sauvant 1992) et deux approches conceptuelles différentes ont émergé pour simuler ces trajectoires, l'une ascendante, l'autre descendante que nous appellerons avec la terminologie anglosaxonne «bottom-up » et «top-down » respectivement. Les progrès réalisés avec ces deux approches de modélisation des trajectoires sont brièvement décrits cidessous. L'approche « bottom-up » considère la trajectoire comme la résultante de changements homéorhétiques (voir encadré) sous-jacents, c'est-à-dire les changements coordonnés génétiquement dans les profils hormonaux (et les affinités des récepteurs associés) observés lorsque les animaux passent d'un état physiologique à un autre (par exemple de la fin de gestation au début de la lactation). En revanche, l'approche «topdown » considère plutôt la trajectoire comme étant un but que l'animal est programmé pour atteindre, c'est-à-dire que la trajectoire est tirée par une finalité elle-même intégrée au génome, ce qui correspond au concept de la téléonomie (Monod 1970). Dans cette perspective, ce sont les trajectoires qui affectent directement l'aptitude de l'animal et sont donc l'objet de la sélection. Bien que ces deux approches diffèrent dans leur base conceptuelle, elles reconnaissent toutes deux que la partition des nutriments est soumise à deux processus distincts : le contrôle génétique et la régulation homéostatique, comme le montre la figure 1. Une grande partie du défi dans la prédiction de la partition des éléments nutritifs est lié à la compréhension et à la représentation du couplage de ces deux aspects et de leur contrôle par le génotype.

\section{2 / L'approche « bottom-up » : la modélisation des trajectoires homéorhétiques}

L'approche «bottom-up » cherche à établir des règles de fonctionnement au niveau des régulateurs métaboliques qui génèrent des trajectoires de rendement au niveau des animaux. Cette approche est toujours celle qui est prise dans les modèles mécanistes métaboliques. Un des premiers exemples de cette approche chez des vaches laitières est le modèle de la glande mammaire de Neal et Thornley (1983) qui a été utilisé par Baldwin et al (1987) pour étendre leur modèle métabolique de la vache laitière, Molly, afin de simuler différentes étapes de la lactation. Dans le modèle de glande mammaire de Neal et Thornley, le potentiel de production de lait de la vache est une fonction du nombre de cellules sécrétrices mammaires qui évolue à son tour au cours de la lactation par l'intermédiaire d'une hormone de lactation théorique. Cela a permis de disposer dans le modèle métabolique Molly d'une trajectoire homéorhétique pour la production de lait qui représente une fonction de demande en nutriments évoluant au cours de la lactation tout en restant indépendante de l'approvisionnement en éléments nutritifs. Par la suite, cette trajectoire homéorhétique pour la production laitière a été affinée en élaborant des descriptions de plus en plus sophistiquées de la prolifération des cellules sécrétrices mammaires et de leur apoptose (Dijkstra et al 1997, Pollott 2000, Vetharaniam et al 2003b). Bien que ces modèles de la glande mammaire incorporent progressivement des descriptions plus mécanistes du nombre de cellules mammaires et de leur fonctionnement, il est intéressant de noter que le facteur déterminant de ces modèles reste essentiellement le temps depuis le vêlage, plutôt que n'importe quelle force motrice génétique innée.

Il est également intéressant de noter que cette trajectoire homéorhétique pour le lait ne fait pas de distinction entre les composants du lait. Dans la majorité des modèles métaboliques mécanistes (Danfaer 1990, Baldwin 1995, Martin et Sauvant 2007), les profils de production potentielle de matières grasses, de protéines et de lactose du lait sont directement liés à la production de lait, ou à la trajectoire du nombre de cellules mammaires et s'appuient ensuite sur la partition des éléments nutritifs pour générer des différences dans la composition du lait. Cette approche a montré ses limites et a conduit à introduire des modifications dans la représentation des profils

Figure 1. Représentation schématique du principal défi pour améliorer la prévision de la partition des nutriments: incorporer la partition liée aux forces motrices dépendant du génome dans les modèles de partition des nutriments conçus pour maintenir l'équilibre physiologique.

La flèche inverse représente le fait que l'environnement nutritionnel peut influer sur l'expression des gènes et donc sur la partition qui est contrôlée par les gènes.

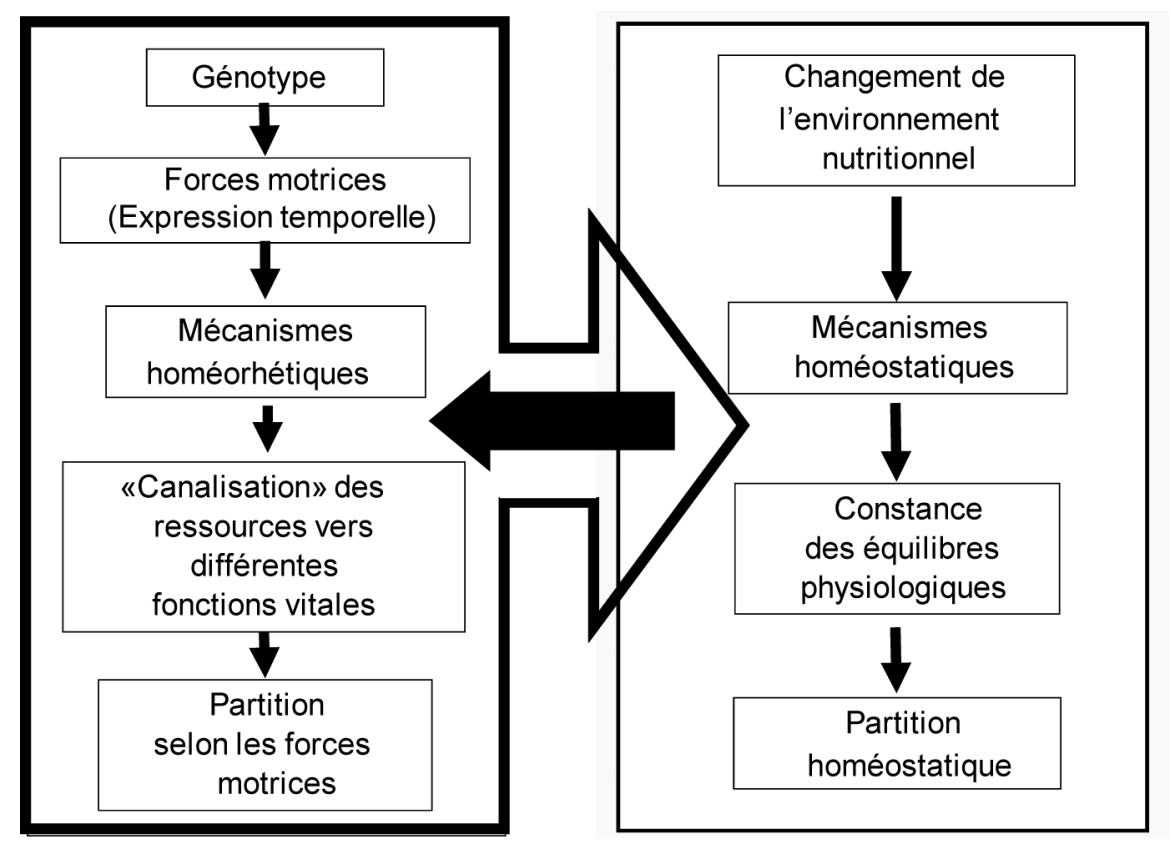


potentiels de composition du lait avec, par exemple, une dissociation de la production de lactose du lait de celles de matières grasses et de protéines (Hanigan et al 2007). Cette adaptation dans laquelle la vitesse maximale de la synthèse du lactose a été modulée en fonction du stade de lactation, tandis que les vitesses maximales de synthèses de matière grasse et de protéines du lait restent fonction $\mathrm{du}$ nombre de cellules mammaires actives, est assez conforme aux réflexions sur la sécrétion de lactose (cf. plus haut) et a sensiblement amélioré la capacité de Molly à prévoir les variations de composition du lait au cours de la lactation. Toutefois, il subsiste des biais de prévision en ce qui concerne la matière grasse du lait en fonction du stade de lactation (Hanigan et al 2007).

De la même façon, il n'existe pas de trajectoires explicites homéorhétiques pour l'utilisation des réserves corporelles dans la majorité des modèles métaboliques, à une exception près (Martin et Sauvant 2007). A titre d'exemple, dans Molly les variations des réserves lipidiques corporelles sont régulées par la taille du pool de glucose rapportée à une valeur de référence. Ainsi, l'accumulation plasmatique de glucose favorise la lipogenèse alors que la pénurie de glucose induit une lipolyse (Baldwin 1995). Le lien entre les concentrations de glucose et les variations des réserves lipidiques est assuré par un signal anabolique et un signal catabolique, critères pertinents assimilables à des «métahormones» qui représentent globalement l'insuline et le glucagon d'une part et les catécholamines d'autre part (Baldwin 1995). Bien que la régulation de la lipolyse et de la lipogenèse par l'intermédiaire des méta-hormones ait été élaborée pour assurer le contrôle de rétroactions plus sophistiquées (Hanigan et al 2009, 2007), il n'existe pas de trajectoire explicite pour les réserves corporelles dans les versions publiées de Molly. Des trajectoires en apparence plus concrètes d'hormones se retrouvent dans le modèle de Danfær (1990), qui utilise, comme force motrice, des évolutions de teneurs moyennes en hormone de croissance et en insuline pour modifier les prélèvements des nutriments par la glande mammaire, le muscle et le tissu adipeux. Dans ce modèle, ces hormones sont affectées par le stade de lactation, ainsi que par la production de lait et le poids vif. Via ces trajectoires, le modèle de Danfær contient un contrôle homéorhétique de la partition des nutriments, bien que ceci ne soit pas explicité ainsi par l'auteur. Le modèle de Sauvant (1994) fait un pas important en rendant explicite le contrôle homéorhétique dans son architecture et met en œuvre cette régulation en utilisant des méta-hormones cataboliques et anaboliques. Cette pre- mière approche a été concrétisée par Martin et Sauvant (2007) afin de pouvoir inclure expressément les changements génétiquement régulés des réserves lipidiques corporelles par une hormone de mobilisation et une hormone de l'anabolisme qui ont été inversement liées pour assurer une corrélation négative, ou une substitution largement exclusive, entre la mobilisation et l'accrétion des lipides corporels. Dans ce modèle les niveaux de ces hormones homéorhétiques sont explicitement fonction du potentiel génétique de production du lait et fournissent ainsi des trajectoires homéorhétiques d'utilisation des réserves corporelles qui sont génétiquement pilotées. Bien que tous ces modèles reconnaissent la nécessité d'avoir des trajectoires génétiquement pilotées (soit explicitement soit implicitement), ils utilisent le temps comme force motrice pour générer les trajectoires homéorhétiques et ne pas invoquer l'expression génétique (ou dépendant d'un état physiologique). Cela peut sembler un détail, mais les études de croissance ont montré que les trajectoires relatives aux mesures de l'état physiologique, par exemple le degré de maturité, élargissent considérablement la capacité des modèles à tenir compte des différences entre génotypes (Taylor 1980, Emmans et Kyriazakis 2001, Doeschl-Wilson et al 2007).

Figure 2. Représentation schématique de l'animal comme un système régulé (d'après Sauvant 1994).

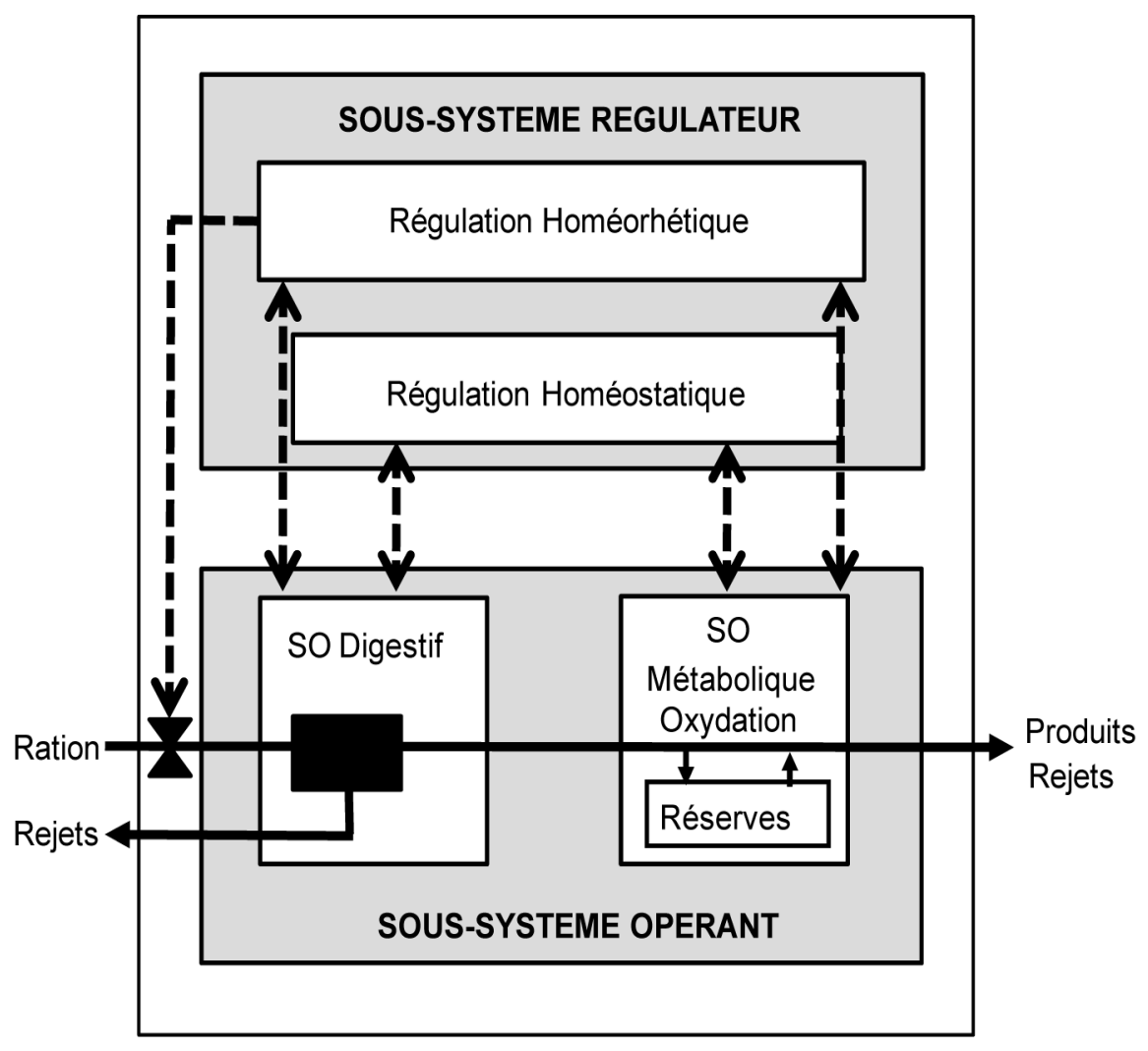

\section{3 / Les approches «top-down » : la modélisation des trajectoires téléonomiques}

L'approche «top-down » vise à définir des règles de fonctionnement au niveau animal dans le but de réguler la partition de nutriments vers les différents processus physiologiques (entretien, lait...). Cette approche est habituellement celle utilisée dans les modèles de flux de nutriments. Elle utilise des phénomènes biologiques observés qui sont décrits en termes de finalités évolutives de l'animal. Cette approche reconnaît explicitement la notion de pilotage génétique des fonctions vitales ; ce pilotage se traduit par un ensemble cohérent de propriétés émergentes d'une multitude de mécanismes sous-jacents médiés par des profils d'expression génique, perfectionnés par la sélection afin de maximiser la probabilité de transmission des gènes à la génération suivante. Compte tenu du fait que l'on considère que ces trajectoires sont orientées en fonction d'une finalité, elles sont qualifiées de téléonomiques (Monod 1970).

La notion de « trajectoires téléonomiques » n'est pas nouvelle. Elle est implicite dans l'idée de courbes de potentiel de croissance ou de lactation, car elle implique un objectif de performance génétiquement programmé. Lorsque l'on crée un stress environnemental, on 
Figure 3. Schéma du cadre conceptuel utilisé pour représenter les priorités des animaux, les transferts et les flux entre celles-ci dans le modèle de Martin et Sauvant (2010a).

Les priorités suivantes sont représentées: G, la croissance ; A, le vieillissement ; $R$, l'équilibre des réserves corporelles ; $U$, la survie du veau à naître ; $N$, la survie du veau nouveau-né ; S, la survie du veau de lait.

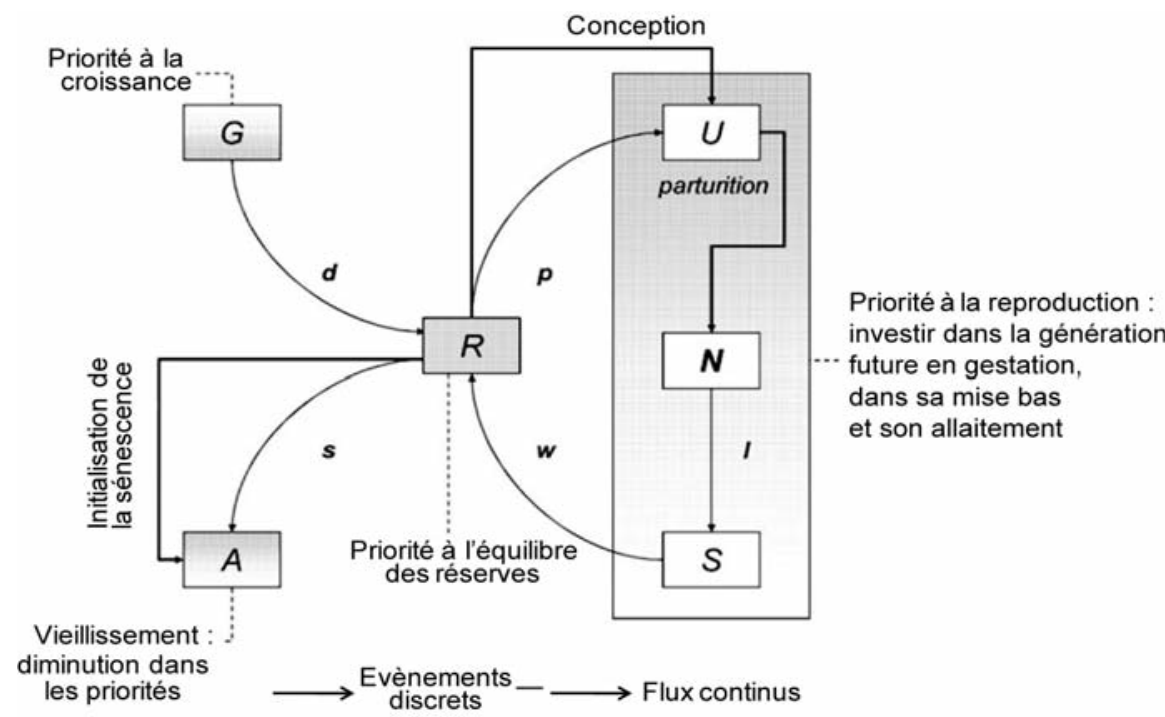

peut temporairement inciter l'animal à dévier de sa trajectoire pour faire face à ce stress, mais la production tend à revenir à sa trajectoire dès que les conditions redeviennent normales. Cette propriété s'observe même si la période difficile est placée en tout début de lactation et dure plusieurs mois (Friggens et al 1998, Delaby et al 2009). Les modèles qui cherchent à décrire le potentiel de croissance ont une longue histoire (Emmans 1997, Taylor 1980) et des modèles de lactation explicitement destinés à décrire le potentiel, ont également été proposés (Friggens et al 1999).

La notion de trajectoires téléonomiques de variation des réserves corporelles a également commencé à émerger il y a quelque temps. Des expériences qui manipulaient la note d'état corporel au vêlage ont montré que les vaches suivaient des trajectoires de changement d'état corporel en début de lactation qui étaient compatibles avec l'idée d'une force motrice visant à atteindre un état corporel cible particulier (Broster et Broster 1998, Garnsworthy et Topps 1982). Ces observations permettent de faire l'hypothèse que chez les vaches laitières, comme chez d'autres mammifères (Pond 1984), les changements de réserves corporelles sont programmés en fonction du statut de reproduction (Friggens 2003). Ces phénomènes ont été formalisés pour fournir un modèle capable de prévoir les trajectoires téléonomiques des variations des lipides corporels pour les vaches laitières en fonction de l'état des réserves au moment du vêlage, du stade de lactation et du stade de gestation (Friggens et al 2004). l'animal. Non seulement l'examen des priorités relatives fournit des explicavie dans le modèle de Martin et Sauvant (2010a). tions fonctionnelles pour des phénomènes tels que la baisse de la fécondité lorsque la production de lait augmente (Friggens et al 2010), mais il fournit également la composante animale d'un cadre capable de prévoir la partition des nutriments. Cela a été admis par Sauvant (1994), qui a proposé une architecture générique d'un organisme basée sur le couplage d'un sous-modèle de régulation fournissant les forces téléonomiques pour piloter le fonctionnement d'un sous-modèle opérant (figure 2), et a été récemment développé dans un modèle téléonomique de la partition des nutriments.

Dans ce modèle téléonomique développé par Martin et Sauvant (2010a, b), le sous-modèle régulateur décrit la dynamique des priorités relatives d'un mammifère femelle entre les fonctions de la vie destinées à la croissance $(G)$, au vieillissement $(\mathrm{A})$, à la gestion des réserves corporelles (R), à l'approvisionnement en nutriments pour le fœtus (U), le nouveau-né (N) et le jeune nourri au lait (S) (figure 3). Ce modèle dynamique (figure 4) constitue le pilote pour la partition des nutriments dans le sous-modèle opérant. Ainsi, ce modèle, paramétré pour des vaches laitières, permet de décrire les modifications du poids vif et de sa composition, la croissance du fœtus, la production laitière et sa composition ainsi que l'ingestion d'aliments des vaches laitières pendant la croissance, pendant plusieurs cycles successifs de reproduction et au cours du vieillissement. Pendant les cycles de reproduction, les priorités relatives ont été construites pour assurer l'évolution et la continuité métabolique entre la

Figure 4. Trajectoires des priorités pour la croissance (G), le vieillissement (A), l'équilibre des réserves corporelles $(R)$, la survie du veau à naître $(U)$, la survie du veau nouveau-né (N), et la survie du veau de lait (S) pendant plus de 1500 jours de

Les flèches indiquent les mises bas lors de deux cycles successifs de reproduction.

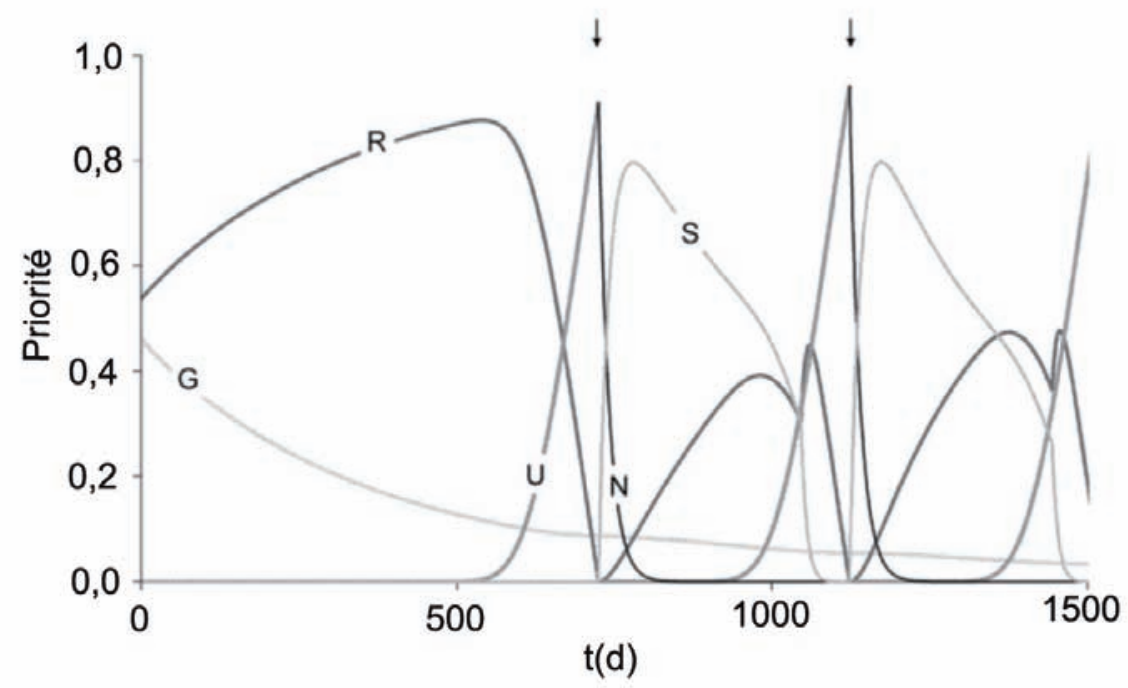


gestation et la lactation, et donc garantir la survie des jeunes, gérant ainsi le compromis entre la priorité d'investir dans le cycle actuel de reproduction et la priorité pour commencer un nouveau cycle de reproduction. L'élasticité des réserves corporelles est considérée comme une composante intégrante du succès de la reproduction : la mobilisation des réserves corporelles lors de la parturition a pour objectif de sauvegarder le cycle de reproduction de la future génération. Elle est donc explicitement considérée comme un investissement maternel pour fournir des éléments nutritifs afin de satisfaire les besoins du veau, la reconstitution des réserves corporelles étant programmée à la fin d'un cycle de reproduction. Le modèle ainsi construit est capable de simuler de façon satisfaisante les grandes variables de production (le poids, la production de lait, l'ingestion) tout au long de la vie productive de la vache, et ce pour différents génotypes bovins (Martin et Sauvant 2010a).

Il existe d'autres modèles de type « topdown » qui reconnaissent explicitement plusieurs forces motrices. Les modèles de Petruzzi et Danfær (2004), BrunLafleur (2011) et Baudracco et al (2012) reconnaissent tous explicitement des changements génétiquement programmés des réserves corporelles. On peut aussi considérer que la notion est devenue assez implicite dans les versions récentes du système d'alimentation américain «Cornell Net Carbohydrate and Protein System » (Tylutki et al 2008) et du système des unités d'encombrement de l'INRA (Faverdin et al 2007) puisque les deux reconnaissent l'intérêt de prévoir des trajectoires d'ingestion en tenant compte de variations programmées des réserves corporelles et d'une courbe de lactation potentielle de production laitière. Dans la plupart de ces modèles de trajectoires téléonomiques, et c'est également le cas avec les trajectoires utilisées dans les modèles homéorhétiques, la force motrice reste le temps écoulé à partir d'un événement donné en matière de reproduction (la conception, la mise bas) plutôt qu'une force motrice génétique. Une exception à cette constatation est l'intégration d'une force motrice liée à la maturité de l'animal pour copiloter les phénomènes de croissance (Martin et Sauvant 2010a).

L'intégration des concepts téléonomiques pour la modélisation de la partition des nutriments a l'avantage d'être plus facile à relier à des objectifs évolutifs. Elle offre donc une représentation de cette partition à un niveau relativement agrégé de régulation de toutes les fonctions. Sa vision finaliste doit être vue comme un moyen de décrire simplement les propriétés émergentes des innombrables changements physiolo- giques entraînés par les dynamiques sousjacentes de l'expression des gènes aux différentes étapes de la vie.

\section{2 / Le défi de l'intégration des informations génétiques sur la partition des nutriments}

Il y a maintenant de nombreux travaux montrant que le génotype affecte la partition des nutriments. Des groupes de vaches qui diffèrent par leur âge ou leur génétique présentent des trajectoires différentes. Si on alimente ces animaux avec le même régime distribué en quantité identique, les performances de production seront différentes, les trajectoires de production laitière les plus élevées induisant vraisemblablement une captation de nutriments par la mamelle supérieure (Brun-Lafleur et al 2010). Ces dernières années, un nombre important d'études comparatives de races, de lignées de sélection ont révélé des différences très significatives dans les modes de production de lait et de l'utilisation des réserves corporelles (Horan et al 2005, Dillon et al 2006, Roche et al 2006, Yan et al 2006, Cutullic et al 2009, Delaby et al 2009). En relation avec ces différences de performances, on observe différents types de profils endocriniens (Drackley et al 2001, Gong et al 2002, Lucy et al 2009). De toute évidence, ces différences zootechniques sont soustendues par des différences dans l'expression des gènes et des profils enzymatiques (Loor et al 2006, SumnerThomson et al 2011).

Toutes ces informations ne peuvent laisser aucun doute sur le fait que le génotype joue un rôle majeur dans la détermination de la partition des éléments nutritifs et par conséquent que tout modèle permettant de prévoir la partition des éléments nutritifs qui ne permet pas de simuler des génotypes différents sera inévitablement limité dans son caractère générique. Compte tenu de cela, on peut supposer que les modèles de partition des nutriments devraient prendre en compte le génotype. A ce jour, ce n'est pas le cas et il y a très peu de modèles qui tentent explicitement de le faire (McNamara et Baldwin 2000, Bryant et al 2005). Il y a deux raisons à cela, la première étant que, jusqu'à récemment, l'objet des modèles de prévision de la partition des éléments nutritifs était surtout de prévoir des réponses à des variations d'apports en nutriments, par différents niveaux d'alimentation ou par des changements de composition des rations. C'est ce qu'on appelle une situation "push» dans laquelle la performance est limitée par l'apport en nutriments et par conséquent le génotype a été considéré comme étant fixé. En effet, la majorité des modèles de partition ne tiennent pas compte d'une régulation possible des apports par l'animal lui-même comme une réponse possible à un déséquilibre nutritionnel, mais considèrent que les quantités ingérées sont une entrée du modèle. Dans le contexte d'un contrôle de la partition génétiquement programmé, le fait d'ignorer les réponses possibles sur l'ingestion d'aliments correspond à une vision incomplète du système (cf. § 3).

La seconde raison pour laquelle un nombre limité de modèles intègre véritablement la notion de génotype est liée à la difficulté de décrire de façon mécaniste les mécanismes opérants liés au génotype et qui expliquent la partition des nutriments. Cela peut sembler surprenant étant donné l'amélioration spectaculaire de notre capacité à caractériser les génotypes aux différents niveaux moléculaires (génome, transcriptome, protéome...) et la richesse de l'information désormais disponible pour les vaches laitières (Rhoads et al 2005, Loor 2010). Cette information devrait constituer la base d'une nouvelle approche « bottom-up » capable d'intégrer de façon mécaniste les rôles des génotypes dans les partitions des nutriments. Toutefois, si l'on considère les modèles métaboliques existants qui prennent déjà en compte les sous-classes de lipides, de glucides et de protéines (par exemple, Baldwin 1995, Martin et Sauvant 2007), on peut facilement imaginer que les nouvelles approches intégratives vont devoir prendre en compte a priori des centaines, voire des milliers, d'informations à différents niveaux (métabolome, protéome, génome...). Décrire ces multiples interactions va être un travail gigantesque nécessitant des moyens considérables.

\section{1 / Intégration des informations génétiques dans les approches " bottom-up "}

L'utilisation des méthodes bioinformatiques et celles de la biologie intégrative vont de toute évidence permettre des progrès importants pour identifier les réseaux de gènes associés à des entités physiologiques spécifiques (Loor 2010). Il a été démontré, dans des cas très limités, que les réseaux métaboliques et leurs gènes de contrôle peuvent non seulement être identifiés, mais aussi en partie quantifiés (Goelzer et al 2008, Goelzer et Fromion 2011). Même à ce niveau sub-cellulaire, une approche systémique, et pas seulement bioinformatique, a été nécessaire afin de simplifier (en identifiant les nœuds d'auto-régulation et de commande) et de structurer le réseau en termes fonctionnels et manipulables (Goelzer et al 2008). Une illustration de ceci a également été donnée par Lemosquet et al (2010) qui a montré que même 
avec des systèmes simplifiés de réactions métaboliques, il existe a priori un nombre infini de combinaisons des différents composants pour obtenir un même résultat net, sauf si des hypothèses précises sont formulées pour définir des critères d'optimisation (par exemple maximiser la production d'ATP). Même si la vision ascendante de la partition des nutriments est plus proche de la réalité du fonctionnement biologique et mérite d'être poursuivie, elle soulève actuellement des difficultés considérables pour intégrer les effets des génotypes à des échelles moléculaires. D'autres approches doivent donc aussi être envisagées pour nous permettre de progresser dans l'intégration des effets génétiques sur la partition des nutriments.

Le travail de Lemosquet et al (2010) met en évidence le fait que de nombreuses variantes de la machinerie métabolique peuvent fonctionner convenablement et qu'il n'est pas possible de distinguer a priori parmi toutes ces voies celles qui sont les plus intéressantes sans avoir défini les critères d'efficience au niveau de la « machinerie métabolique ». En d'autres termes, en s'inspirant de ce principe, la prévision de la partition des nutriments en fonction du génotype va exiger que les différences dans le génotype puissent être traduites en termes de fonction objectif appliquée au système considéré. Bien que commune avec le domaine de l'écologie (Yearsley et al 2001), la notion de modèles nutritionnels incorporant une finalité exprimée à travers un critère d'optimisation est rare dans le domaine des sciences animales. Une exception concerne les travaux de Tolkamp et Ketelaars (1992) qui ont suggéré que l'animal en croissance cherchait à optimiser l'efficience d'une telle fonction. Dans ce contexte, l'approche «topdown " semble être la plus capable de fournir des descriptions opérationnelles du génotype pour prédire la partition des nutriments.

\section{2 / Intégration des informations génétiques dans les approches «top-down »}

Dans l'approche «top-down » de la prévision de la partition des nutriments il est relativement facile d'intégrer la notion de génotype. Ainsi, Martin et Sauvant (2010a) ont intégré dans leur modèle de priorités relatives, des paramètres génétiques simples qui peuvent permettre de simuler des trajectoires de performances potentielles de production laitière et des réserves corporelles pour des vaches de valeur génétique élevée et faible. Pour la production de lait, il y avait déjà eu plusieurs modèles d'objectifs comparables (Neal et Thornley 1983b, Ostergaard et al 2000, Vetharaniam et al 2003b). En outre, McNamara et Baldwin
(2000) ont proposé la façon dont le modèle Molly pourrait en principe intégrer différents génotypes en ajustant les taux de la synthèse des matières grasses du lait et du lactose. Actuellement, il existe peu de modèles qui intègrent explicitement les effets du génotype sur de multiples fonctions (effets pléiotropiques) ou sur la partition des nutriments (Bryant et al 2008, Martin et Sauvant 2010a). Enfin, bien qu'il soit relativement facile d'intégrer conceptuellement un effet génotype, il n'est pas aisé d'obtenir des représentations opérationnelles de l'effet du génotype sur la partition des nutriments, la principale question étant celle du lien entre les concepts et les mesures obtenues sur les animaux.

Les évaluations génétiques basées sur des jours-contrôles de mesures de la production de lait (visites mensuelles en général du contrôle laitier dans les exploitations) ont longtemps été utilisées, en combinaison avec les informations généalogiques, pour calculer des valeurs génétiques de production laitière. Elles ont montré leur efficacité et elles ont été plus récemment étendues afin d'inclure d'autres mesures (par exemple la note d'état corporel, Berry et al 2002). Ces dernières fournissent des estimations des variations phénotypiques de la production de lait et de la note d'état corporel qui peuvent être attribuées au génotype. Ces approches ont été appliquées pour fournir des profils de lactation pour les différents génotypes (Veerkamp et Goddard 1998, Coffey et al 2001, Coffey et al 2003, Berry et al 2006). Il est important de noter que ces trajectoires « génétiques » ne sont pas de véritables trajectoires génétiques potentielles. En effet, les modèles statistiques utilisés pour estimer ces trajectoires prennent en compte des effets liés aux exploitations agricoles afin de refléter la moyenne de la population. En conséquence, si les vaches ne sont pas alimentées pour exprimer leur potentiel à un stade de lactation donné, alors un biais consécutif apparaîtra dans les courbes moyennes du génotype.

Néanmoins, il semble raisonnable de supposer que ces courbes reflètent en moyenne le potentiel pour des systèmes d'alimentation couramment pratiqués et, en faisant cette hypothèse, il devient possible d'intégrer de l'information sur le génotype, ainsi estimée, dans les modèles de la partition des nutriments (BrunLafleur 2011). Ainsi, dans le modèle de lactation de Brun-Lafleur (2011), l'information génétique a été construite en utilisant une partie du modèle d'évaluation génétique des caractères laitiers à partir des performances élémentaires (Leclerc et al 2008). Cela permet de simuler la valeur attendue de la production et de la composition du lait dans des conditions d'alimentation moyennes françaises. Cette valeur dépend des caractéristiques de la vache (race, numéro de lactation, stades de lactation et de gestation) ajustées pour tenir compte des facteurs tels que l'âge et le mois au moment du vêlage et la durée de la période de tarissement, comme cela se fait dans le contrôle laitier. En utilisant ces valeurs issues du modèle d'évaluation génétique en même temps que le contexte nutritionnel de la vache (apports en protéines et énergie de la ration), le modèle calcule la production de lait et sa composition réelle. Les résultats obtenus sont suffisamment précis pour permettre d'élaborer la base d'un modèle de production au niveau du troupeau (Brun-Lafleur 2011). Ils fournissent également la preuve de l'intérêt d'estimer les paramètres des rendements potentiels spécifiques à un génotype donné (Bryant et al 2007).

Bien que cette approche de la représentation du génotype n'ait pas encore été explicitement faite pour les trajectoires téléonomiques de la partition des nutriments, il sera assez facile de le faire, en particulier pour la partition de l'énergie. Les courbes génétiques d'état et de composition corporelle au cours de la lactation (Banos et al 2005, Banos et Coffey 2010) correspondent à des courbes de cumul des variations du bilan d'énergie. De ce fait, ces courbes reflètent des conditions d'alimentation moyennes et peuvent être considérées comme des mesures des différences entre génotypes relatives à la partition de l'énergie. Ainsi, il semble vraisemblable que dans un avenir proche la prédiction des trajectoires de partition des nutriments chez la vache laitière sera en mesure d'intégrer des données opérationnelles pour décrire les différences génotypiques. Il s'agira d'une étape vers une pleine intégration du génotype.

\section{3 / Les effets génétiques sur la capacité homéostatique}

L'étude des interactions génotype $x$ environnement (Bryant et al 2006, Beerda et al 2007) montre qu'il existe une composante génétique à la capacité de l'animal à faire face à des «défis » (challenges) nutritionnels plus ou moins soudains. Ainsi, un modèle ayant l'ambition de prévoir les effets du génotype et de l'environnement sur la partition des nutriments ne peut ignorer les effets génétiques sur la capacité homéostatique des individus. La capacité de l'animal à répondre aux perturbations externes par des mécanismes de réponse homéostatiques pour retrouver un état d'équilibre physiologique est essentielle à sa survie. Chez les ruminants, le tube digestif, en particulier le rumen joue un rôle important dans l'homéostase et la robustesse de l'ensemble de l'organisme, mais ces aspects ne seront pas abordés ici. 
L'homéostase métabolique énergétique a été assez bien étudiée et a été en partie représentée dans les modèles métaboliques (Martin et Sauvant 2007). Nous savons qu'il y a des limites dans la capacité d'un animal à gérer son homéostasie. Ainsi, le fait de placer des vaches laitières très fortes productrices dans des environnements nutritionnels défavorables autour du vêlage peut induire des dysfonctionnements métaboliques (Ingvartsen 2006). A ce jour, ce qui a été moins étudié, concerne le lien entre la capacité homéostatique, les trajectoires génétiquement programmées et le génotype, particulièrement vis-à-vis de la partition des nutriments. La capacité homéostatique de la vache laitière est-elle la même pendant toute la lactation ? Cette capacité homéostatique de la vache laitière est-elle affectée par le génotype ?

De plus en plus de résultats montrent que la sélection accrue pour la production laitière augmente le recours à la mobilisation des réserves et réduit la capacité d'adaptation, ou de robustesse, des vaches laitières. Dans un essai étudiant l'interaction alimentation $\times$ race, Delaby et al (2009) ont constaté que les vaches de race Holstein avaient perdu plus d'état corporel et étaient plus maigres au minimum de la courbe de la note d'état, que celles de la race Normande indépendamment de l'alimentation. En outre, la différence de perte d'état corporel entre les régimes alimentaires haut et bas était également plus grande pour les vaches Holstein (Delaby et al 2009). Dans un même ordre d'idées, des différences significatives dans la partition des nutriments ont été trouvées dans un essai comparant 2 lignées de Holstein néo-zélandaises (sélection des années 1970 et 1990) et une lignée Holstein d'Amérique du Nord (1990) (Lucy et al 2009). Dans cette étude, la souche nord-américaine a produit plus de lait et a plus mobilisé les réserves corporelles que les vaches sélectionnées en Nouvelle-Zélande en 1990, ces dernières ayant produit plus que les vaches issues de la lignée sélectionnée en 1970. Cette étude a également montré que ces différences dans la partition des nutriments ont été vraisemblablement sous-tendues par un découplage beaucoup plus important de l'axe somatotrope (GH IGF-1) dans la lignée nordaméricaine par rapport à la lignée néo-zélandaise des années 1970, la lignée de 1990 étant intermédiaire. Une augmentation des apports énergétiques n'a eu aucun effet sur la production laitière des Holstein nordaméricaines en début de lactation, alors qu'elle a induit une augmentation de la production de lait dans la lignée néo-zélandaise de 1970 (Grala et al 2011).

\section{1 / Différences de capacité homéostatique pour des effets «push » et «pull»}

Les résultats de ces comparaisons entre lignées d'une même race suggèrent que la sélection pour accroître la production laitière a vraisemblablement réduit la marge de manœuvre en matière de sous alimentation énergétique. Ce constat a été confirmé par une étude sur les effets combinés de la qualité de la ration et la fréquence de traite chez des vaches de potentiels génétiques différents (Beerda et al 2007). Dans cette étude, deux groupes de vaches de valeur génétique élevée $(H)$ et faible (L) pour la production laitière ont été comparés. L'effet d'un challenge nutritionnel sur ces deux groupes génétiques a été évalué en comparant une ration semi-complète ( $8 \mathrm{~kg}$ de concentrés) de forte densité énergétique (E) avec une ration semi-complète à forte proportion d'ensilage d'herbe et faible densité énergétique (3 $\mathrm{kg}$ de concentrés) (S). La réponse aux changements dans la fréquence de traite a également été évaluée en comparant 2 et 3 traites par jour avec les deux régimes. Comme prévu, les vaches du groupe $\mathrm{H}$ produisent plus de lait que les vaches laitières du groupe L avec 2 traites par jour. En outre, la diminution de production de lait à $4 \%$ de matières grasses associée à l'alimentation $\mathrm{S}$ est plus grande avec les vaches du groupe $\mathrm{H}$ (figure 5). A partir de ce résultat, il est tentant de conclure que la production de lait de vaches fortes productrices est plus sensible à un stress nutritionnel que celle de vaches dont le potentiel génétique est plus faible. Toutefois, si l'on compare les deux fréquences de traites avec le régime $E$ riche en énergie, le résultat inverse est obtenu, la production de lait des vaches à faible valeur génétique (L) est plus sensible au nombre de traites quotidiennes que celle des vaches à forte valeur génétique $(\mathrm{H})$. De toute évidence, la production de lait des différents génotypes ne répond pas de façon identique aux différents types de challenge. Biologiquement parlant, la fréquence de traite a plutôt un effet " pull » sur la production laitière, c'est à dire limitant l'effet d'une charge supplémentaire de lait, et empiétant ainsi sur les mécanismes par lesquels la vache ajuste son investissement pour le veau. Alors que le challenge alimentaire a plutôt un effet «push »sur la production laitière, imposant les flux de nutriments du tractus gastro-intestinal vers la glande mammaire. Lorsque toutes les combinaisons de traitements sont prises en considération, cette dichotomie «push»/ «pull» devient plus claire (figure 5). Tous les groupes répondent positivement à un apport d'énergie supplémentaire (effet «push»), mais ceux qui ont une demande supplémentaire pour produire (effet «pull» supérieur), en raison d'une meilleure valeur génétique ou d'une fréquence de traite plus élevée, répondent plus fortement. Toutefois, il convient de noter que les effets de la valeur génétique et de la fréquence de traite ne sont pas additifs vis-à-vis de la production de lait à $4 \%$. En ce qui concerne le bilan énergétique, les vaches faibles productrices ont une plus grande capacité à répondre à un challenge lié à la fréquence de traite, bien qu'elles mobilisent généralement moins sur un traitement donné. Il semble donc que la sélection des vaches

Figure 5. Effet du génotype et de la fréquence de traite sur les réponses de la production de lait $(A)$ et sur le bilan énergétique $(B)$ selon la densité énergétique de la ration (riche en ensilage (S), riche en concentrés et en énergie $(E))$. D'après les résultats de Beerda et al (2007).

Les réponses des animaux à fort index laitier sont indiquées par des cercles, celles des animaux à faible index laitier par des triangles. Les réponses liées à une fréquence de 2 traites par jour sont indiquées en noir, et celles liées à une fréquence de 3 traites par jour en rouge.

A

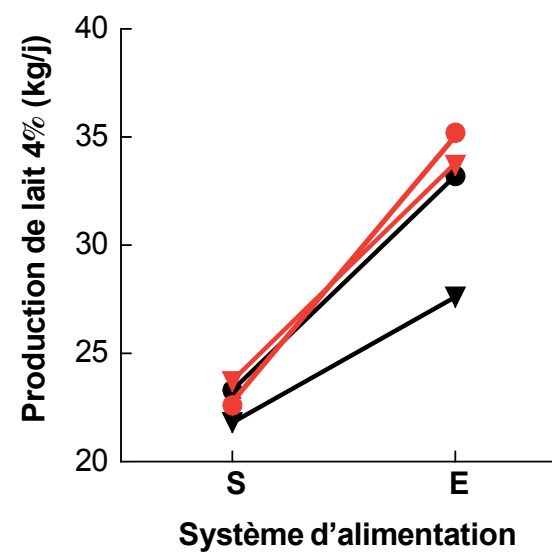

B

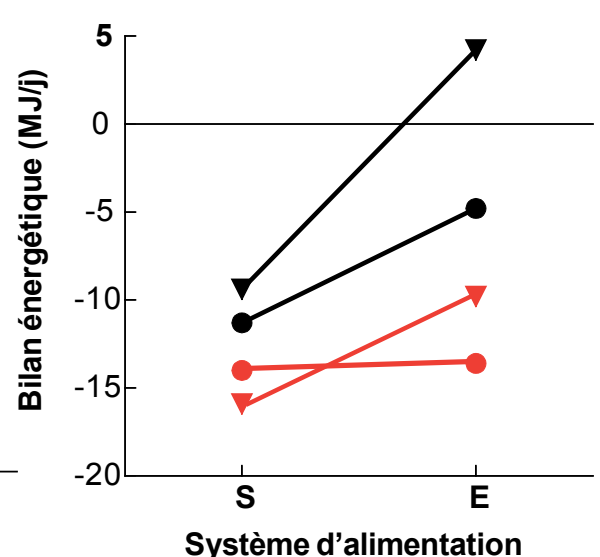


pour un caractère spécifique de production diminue leur robustesse, ce qui est confirmé par des études dans d'autres espèces (Reznick et al 2000, Theilgaard et al 2007) et aussi par des travaux de simulation (van der Waaij 2004). Ainsi, la sélection continue pour accroître la production laitière n'est pas sans conséquence sur les autres fonctions (voir aussi Friggens et al (2010)), contrairement à ce que prétendent certains auteurs (Collier et al 2005).

\section{2 / La prise en compte de la capacité homéostatique dans les modèles}

À l'heure actuelle, les connaissances concernant le rôle du génotype et du stade de lactation sur la capacité homéostatique des animaux n'ont pas été explicitement incluses dans les modèles métaboliques qui permettent de prévoir la partition des nutriments. Bien que Vetharaniam et al (2003a) aient modélisé avec succès les effets de la fréquence de traite et des apports en énergie sur les populations de cellules sécrétrices de la glande mammaire et l'aptitude à produire du lait, c'était plus l'effet de facteurs environnementaux sur la trajectoire homéorhétique du nombre de cellules mammaires actives qui a été modélisé que la capacité homéostatique de la glande mammaire elle-même. Toutefois, si l'on considère que le partage vers et à partir du tissu adipeux est un élément clé de la réponse homéostatique à des perturbations nutritionnelles, la facilité de mobilisation des lipides corporels selon le stade de lactation est modulée dans certains modèles. En effet dans le modèle de Martin et Sauvant (2007), la sensibilité de l'anabolisme et du catabolisme des lipides du tissu adipeux est une fonction de $l^{\prime}$ « hormone de mobilisation » qui décroît en fonction du stade de lactation. Ainsi, on peut considérer que la capacité homéostatique des réserves lipidiques évolue avec le stade de lactation. L'idée que capacité d'homéostasie varie selon le stade physiologique de l'animal a été formalisée plus précisément dans le modèle de Martin et Sauvant (2010b). Dans ce modèle, la partition de l'énergie vers la production de lait n'est pas seulement une fonction de l'apport réel d'énergie par rapport à l'apport nécessaire, mais elle est aussi fonction de la taille des réserves lipidiques corporelles, et ces fonctions sont modulées par la priorité accordée à la lactation. Cela signifie donc que l'intensité avec laquelle les différentes fonctions vitales d'un organisme sont préservées des perturbations nutritionnelles serait proportionnelle à l'importance relative de cette fonction à ce moment-là.

Il est intéressant de constater que dans les deux modèles évoqués ci-dessus, ces adaptations de la partition en éléments nutritifs sont également fonction de la taille des réserves lipidiques corporelles (Martin et Sauvant 2007, 2010b). Cette fonctionnalité a également été mise en œuvre dans les versions récentes de Molly (Hanigan et al 2007), où l'hormone de lactation qui sous-tend le potentiel de la production de lait interagit également avec les réserves corporelles (par rapport à une valeur de référence équivalente à une note d'état de 3 ), ce que l'on retrouve dans d'autres modèles (Brun-Lafleur 2011). Ce formalisme peut être interprété d'un point de vue très pragmatique, il constitue un facteur d'équilibre dans les modèles de flux métaboliques ou de nutriments. On constate depuis longtemps que les petites erreurs systématiques dans la prévision des flux d'énergie par les modèles « bottom-up » s'accumulent et conduisent parfois à des estimations irréalistes des variations des réserves corporelles sur de longues périodes comme une lactation (McNamara 2004). Ce biais peut être réduit en intégrant une rétroaction de la taille des réserves lipidiques pour réguler la parti-

Figure 6. Deux exemples de relation entre le taux de substitution fourrage-concentré et la partition de l'énergie (A : d'après Faverdin et al $1991 ; B$ : d'après Friggens et al 1998).

Le graphe A montre la relation entre le taux de substitution et le bilan énergétique de la vache. Les couples de points reliés par une ligne sont des moyennes des mesures effectuées sur les mêmes vaches à différentes époques. Les concentrés utilisés étaient des céréales (carrés), de la pulpe de betterave (cercles) ou du son de blé (triangles). Les fourrages utilisés étaient de l'ensilage de maïs (traits pleins), de l'ensilage d'herbe (tirets) ou du foin (pointillés). La ligne épaisse rouge montre la relation générale.

Le graphe B montre l'évolution du taux de substitution (cercles pleins et tendance en pointillés) avec les semaines après le vêlage. Les taux de substitution sont calculés à partir des différences de consommation (lignes continues) entre deux régimes alimentaires qui ne différaient que par leur teneur en concentrés.
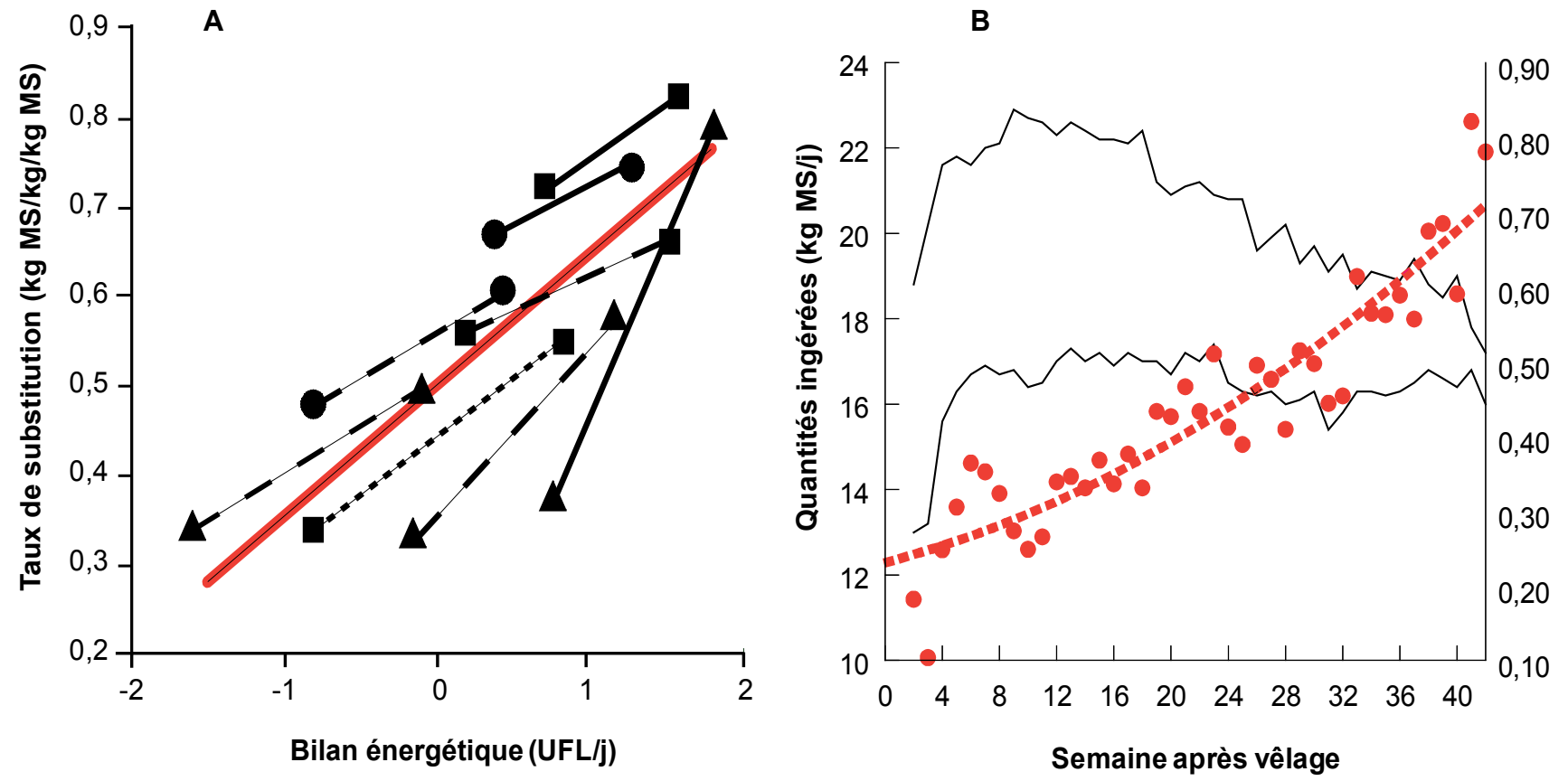

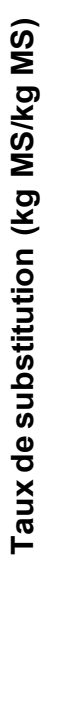


tion de l'énergie vers le lait (Hanigan et al 2007). Dans les modèles qui prévoient l'ingestion d'aliments, la régulation de l'ingestion en fonction du niveau des réserves lipidiques joue globalement le même effet (Martin et Sauvant 2010b), Brun-Lafleur 2011).

\section{3 / Le rôle des réserves corpo- relles et de l'ingestion dans la capacité homéostatique}

En plus de cette interprétation très pragmatique, il y a une explication biologique possible basée sur la théorie lipostatique de la régulation des flux d'énergie (Kennedy 1953, Kennedy 1967, Shalloo et al 2004). Cette théorie, basée sur le fait que les animaux régulent leur ingestion pour maintenir un point d'équilibre des réserves corporelles, a été validée par la découverte de la leptine comme signal du niveau d'adiposité, bien que ce ne soit qu'une partie du système de régulation (Faverdin et Bareille 1999, Chilliard et al 2000). La théorie lipostatique implique que l'un des objectifs de l'animal est de maintenir un niveau cible des réserves corporelles. Kennedy (1967) a admis que ce niveau cible pouvait varier en fonction de l'état physiologique. Dans ce contexte, la découverte de l'action de la leptine sur la reproduction (Hoggard et al 1998) n'est pas surprenante. Cette régulation de l'utilisation des lipides corporels dans les modèles qui cherchent à prévoir la partition des nutriments n'est pas en soi susceptible de fournir des informations utiles pour ajuster la capacité homéostatique au génotype. Cependant, elle met l'accent sur le principe d'une trajectoire téléonomique des réserves cor- porelles. Elle met également en lumière un aspect souvent négligé de la partition des nutriments qui est la régulation de l'ingestion en fonction de trajectoires génétiquement déterminées. Il existe de solides preuves que la régulation négative de l'ingestion en début de lactation par l'utilisation des réserves est, au moins en partie, une régulation qui prend en compte la mobilisation génétiquement contrôlée des réserves corporelles (Bareille et al 1997, Faverdin et Bareille 1999, Friggens et al 2007).

Par ailleurs, le génotype et le stade de lactation jouent un rôle sur la possibilité d'accroître l'ingestion d'énergie en apportant plus d'aliments concentrés. Cette possibilité peut se mesurer par le taux de substitution entre fourrages et concentrés, lequel dépend majoritairement du statut énergétique de l'animal. Ce taux de substitution augmente avec le stade de lactation, c'est-à-dire lorsque la proportion d'énergie supplémentaire apportée et utilisée par la mamelle décroît (figure 6). La forte corrélation négative entre le taux de substitution et la réponse de la production de lait (Faverdin et al 1991) est également observée entre les lignées Holstein sélectionnées sur différents potentiels de production (Horan et al 2006). Si l'on comprend bien qu'avec un taux de substitution plus élevé, le supplément d'énergie ingéré est plus faible et donc que la réponse laitière décroît, il est plus étonnant de constater que le supplément d'énergie lié à l'apport d'aliments concentrés sert de moins en moins à fabriquer du lait (figure 7). Ainsi, plus l'animal semble avoir du mal à écouler l'énergie supplémentaire ingérée, plus il régule les entrées. Ces résultats mon- trent clairement que la partition des nutriments ne peut pas être considérée indépendamment des réponses de l'ingestion en éléments nutritifs.

En ce qui concerne les effets du stade physiologique sur la capacité homéostatique, de nouvelles informations commencent à émerger des études très récentes qui ont réalisé des variations d'apports nutritionnels à différents stades de lactation chez des vaches laitières (BjerreHarpoth et al 2012) et des chèvres (Schmidely et al 2011). Dans les deux espèces, les réponses de la production laitière à une courte période de sous-alimentation sont pratiquement proportionnelles au niveau de production laitière des animaux immédiatement avant le test, sans aucun effet supplémentaire du stade de lactation. Si cela était démontré dans une large gamme de situations, il serait possible de modéliser simplement la capacité homéostatique en fonction du stade de lactation et du génotype en l'exprimant proportionnellement au potentiel de production. Ces observations expérimentales cadrent bien avec les approches adoptées dans certains modèles empiriques récents qui permettent de prévoir les conséquences des changements nutritionnels sur les réponses marginales de production (Brun-Lafleur et al 2010, Maxin 2011). L'ajustement des réponses marginales observées en fonction des niveaux initiaux de performance permet à ces deux modèles de se montrer robustes dans la prévision des réponses relatives de production et de composition du lait. Il semble également probable que les prévisions puissent être encore améliorées en les exprimant proportionnellement à la production (Brun-Lafleur 2011).

Figure 7. Relation entre le taux de substitution et la partition de l'énergie.

Le graphe A montre que les évolutions des taux de substitution et de la réponse de production de lait sont très liées aussi bien lorsque l'on change de régime (Faverdin et al 1991) que lorsque l'on change de type génétique (Horan et al 2006). La conversion de cette relation en termes de partition d'énergie montre sur le graphe B que lorsque le taux substitution devient important $(>0,5)$, le supplément d'énergie apporté par le concentré va de moins en moins vers la glande mammaire.

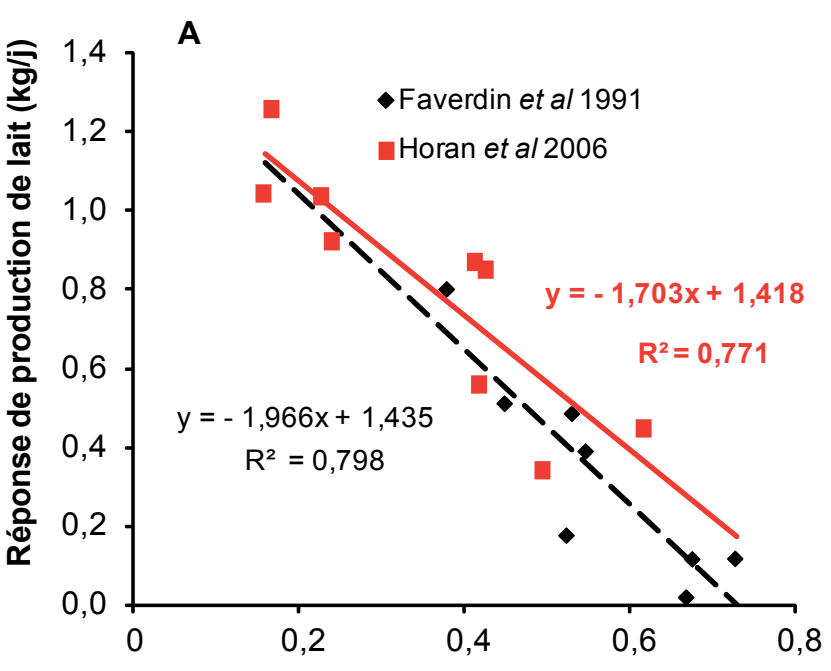

Taux de substitution (kg MS/kg MS)

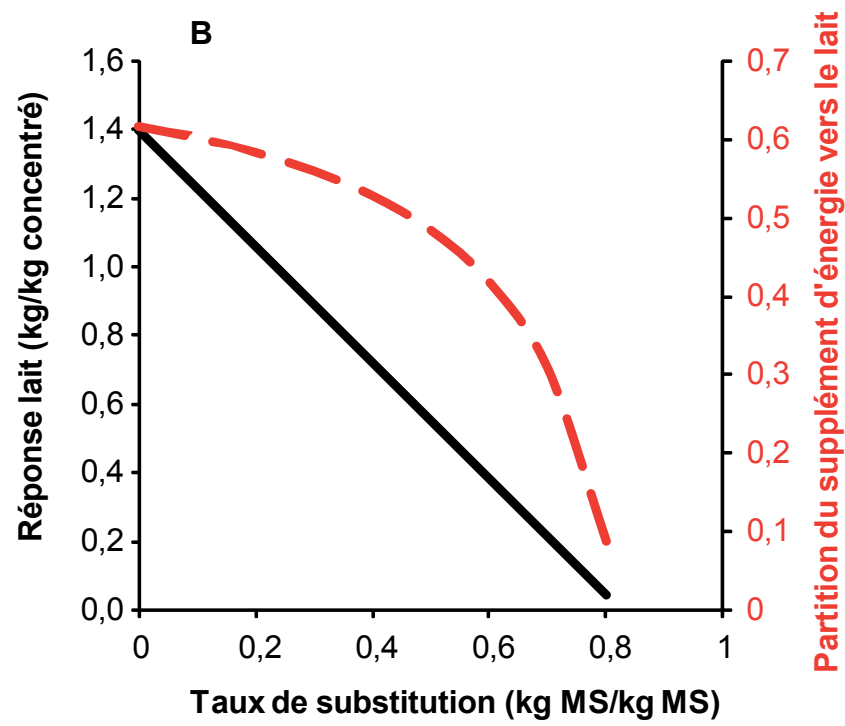

Taux de substitution (kg MS/kg MS) 


\section{4 / Conclusions et perspec- tives}

\section{1 / De nouveaux outils pour le conseil en élevage}

Au cours des 10 dernières années, il est apparu de plus en plus nécessaire d'intégrer dans les modèles nutritionnels des forces motrices internes et « innées » qui modifient la partition des nutriments en fonction de l'état physiologique : les trajectoires génétiquement contrôlées. Dans le même temps, les modélisateurs ont commencé à intégrer dans les modèles des effets génétiques sur ces trajectoires et sur la capacité homéostatique de l'animal. Cela a impliqué la prise en compte de notions telles que la téléonomie, l'homéorhèse et l'homéostasie, afin d'établir les priorités et les trajectoires potentielles des différentes fonctions vitales d'un organisme. En conséquence, des modèles déjà existants ont évolué afin de mieux tenir compte de ces facteurs liés aux animaux. En outre, il y a eu l'émergence récente de nouveaux modèles capables de prévoir la partition des nutriments tout au long de la lactation pour des vaches de différents potentiels de production de lait. A ce jour, ces modèles restent des modèles de recherche, bien que des progrès substantiels aient été accomplis pour élaborer des modèles qui pourront être utilisés en pratique. Dans ce contexte, il semble important de pouvoir développer des modèles stochastiques de partition des nutriments (Brun-Lafleur 2011), l'intérêt des modèles stochastiques pour simuler les stratégies de conduites à l'échelle du troupeau ayant déjà été démontré (Ostergaard et al 2000).

Pour illustrer les potentialités nouvelles de ces approches, le modèle de BrunLafleur (2011) a été utilisé pour simuler les conséquences de conduites alimentaires différentes de vaches laitières au cours de leur lactation sur les données de production, de consommation et de note d'état corporel. Le dispositif simulé était celui d'un essai de long terme conduit à l'INRA du Pin-au-Haras (Delaby et al 2009) au cours duquel les vaches ont reçu des régimes variant par leur densité énergétique, Haut $(\mathrm{H})$ et Bas (B), suivant 4 séquences, $\mathrm{H}-\mathrm{H}, \mathrm{H}-$ $\mathrm{B}, \mathrm{B}-\mathrm{H}$ et B-B. Les vaches étaient en vêlages groupés. Les premiers niveaux $\mathrm{H}$ et $\mathrm{B}$ étaient obtenus en phase hivernale avec deux régimes conservés (ration complète avec $65 \%$ d'ensilage de maïs, $5 \%$ de foin et $30 \%$ de concentrés vs ration avec $45 \%$ d'ensilage d'herbe, $40 \%$ de foin et $15 \%$ de concentrés). Après la mise à l'herbe, les deux niveaux d'apports $\mathrm{H}$ et $\mathrm{B}$ étaient réalisés par des apports différents d'aliments concentrés au pâturage $(4$ ou $0 \mathrm{~kg} / \mathrm{j})$. Ces rations ont été

Figure 8. Exemple de simulation par le modèle SITEL de Brun-Lafleur (2011) des données de production, d'ingestion et de note d'état corporel au cours d'une lactation entière de vaches laitières placées dans le cadre expérimental d'un essai long terme d'alimentation (données de Delaby et al 2009).

Dans ce dispositif, les vaches en vêlages groupés reçoivent successivement des régimes avec une densité énergétique Haute $(H)$ ou Basse $(B)$ suivant 4 séquences $\mathrm{H}-\mathrm{H}, \mathrm{H}-\mathrm{B}, \mathrm{B}-\mathrm{H}, \mathrm{B}-\mathrm{B}$. Pour chacune des séquences le changement d'alimentation intervient au $127^{\mathrm{e}}$ jour de lactation.

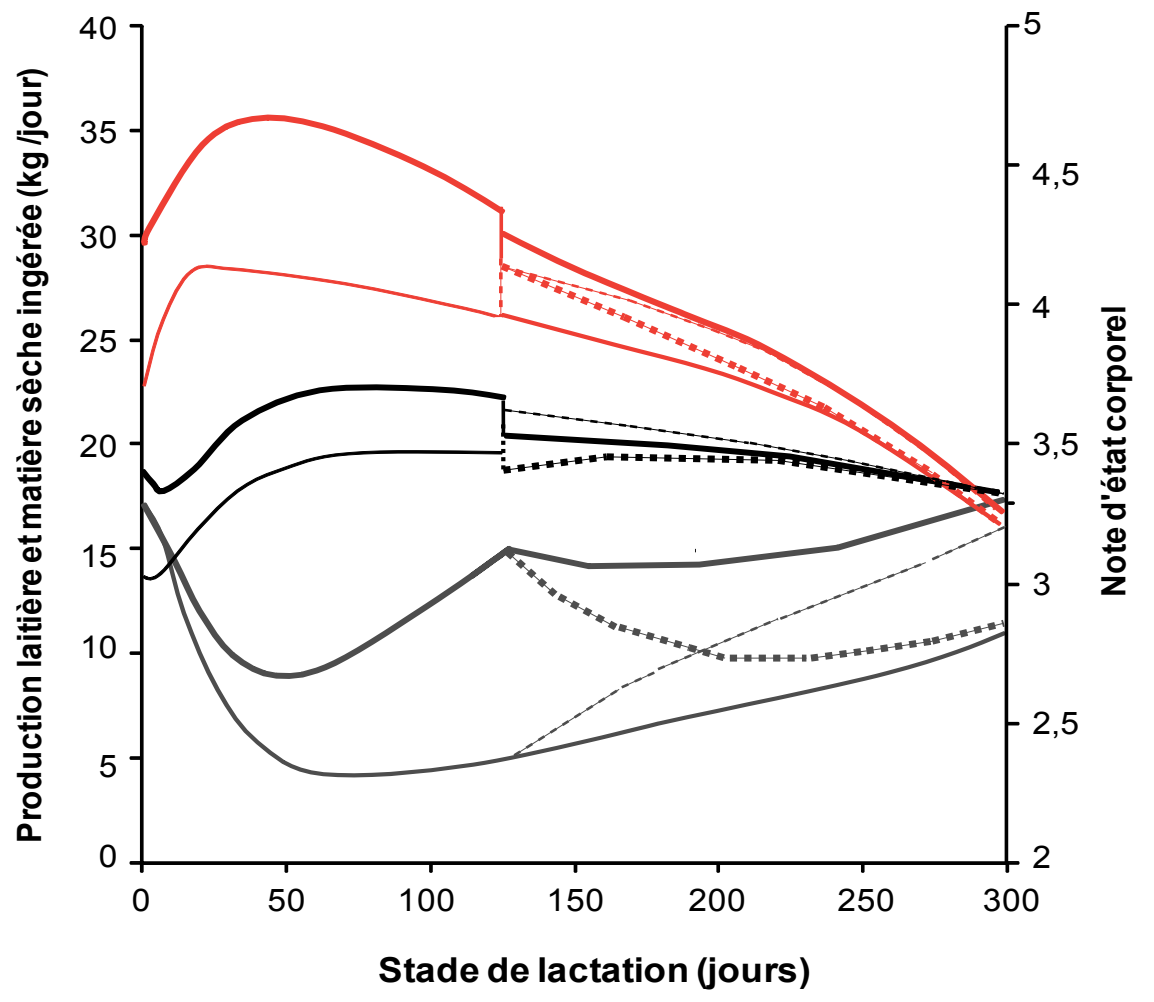

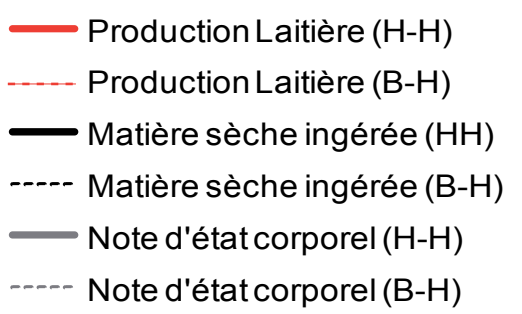

simulées dans la figure 8 pour 4 vaches ayant les mêmes potentialités génétiques et vêlant en même temps. Avec uniquement un potentiel génétique et une description des séquences alimentaires, le modèle reproduit simultanément le comportement des principales variables de la lactation avec une amplitude et une dynamique très voisine de celle des données expérimentales. Ainsi, avec la séquence B-H, la lactation est loin d'exprimer le potentiel de la vache en début de lactation sans que cela handicape la production en seconde partie de lactation avec une alimentation favorable, comme le démontrent les résultats expérimentaux. Le modèle peut également simuler ces mêmes réponses avec des potentiels génétiques différents. De plus, couplé à un autre modèle permettant simuler la réussite des différentes étapes de la fonction de reproduction selon l'état nutritionnel (bilans énergé-

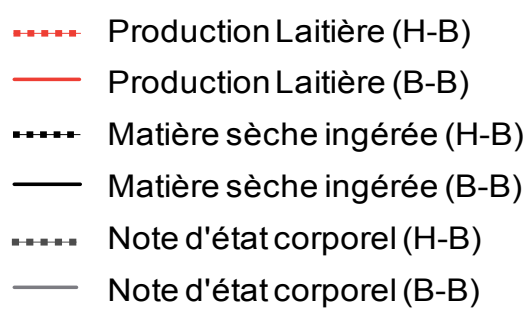

tiques et états des réserves corporelles), il est possible de simuler le lien entre les fonctions de production et de reproduction (Brun-Lafleur et al 2013).

Ces modèles ouvrent la voie à de nouveaux outils de conseil en élevage particulièrement importants pour aborder les notions de « flexisécurité ». Les élevages se trouvant de plus en plus confrontés à des changements rapides des contextes économique et climatique, ils devront reconsidérer les choix de conduite de façon beaucoup plus fréquente que par le passé. De plus, les différents modes de contractualisation des producteurs laitiers avec leurs laiteries diversifient les modes de paiement du lait et les formes d'engagement pour la livraison du lait, ce qui nécessite un conseil plus individualisé et prenant en compte ces différents contextes. Il va donc être essentiel de disposer d'outils permettant 
de simuler la réponse d'un troupeau laitier à différents changements de conduite (alimentation, reproduction, renouvellement...) pour mieux gérer les stocks d'aliments, évaluer les stratégies les plus rentables économiquement tout en vérifiant qu'elles ne font pas courir de risques importants dans un futur plus lointain. Le modèle troupeau de BrunLafleur (2011) est ainsi en cours d'adaptation avec les organismes de conseil en élevage (projet « Preliv ») pour développer un outil de conseil sur la prévision de livraison de lait d'un troupeau en fonction des conduites d'élevage. Cette première tentative permettra donc de mesurer l'apport des travaux sur la partition des nutriments et leur modélisation, sur l'aide à la décision des éleveurs pour optimiser la conduite de leur élevage.

\section{2 / De nombreux défis scienti- fiques}

Ces développements récents ont considérablement étendu la généricité des modèles de partition des nutriments en fonction du type d'animal et de son état physiologique. Cependant, ces modèles restent très largement focalisés uniquement sur la partition entre la production de lait et les réserves corporelles. Le défi qui consiste à relier la prévision de la partition des nutriments à ses conséquences sur la santé, la reproduction et la longévité commence seulement à être abordé. Il s'agit là d'une perspective importante pour les travaux futurs sur la partition des nutriments. Ce défi nécessitera, par exemple, de mettre en cohérence, au sein d'un modèle, les niveaux de détail de la description de la partition des nutriments et de la fonction de reproduction. Ceci exigera également que le système de contrôle (les forces motrices génétiques) soit conçu pour englober les deux modules. En ce qui concerne la reproduction, la compréhension des liens biologiques semble suffisante et il est possible que l'on dispose de suffisamment de données pour progresser dans des délais raisonnables. La tâche sera vraisemblablement plus difficile en matière de santé, en particulier en relation avec la longévité.

Une autre perspective importante sera de pouvoir incorporer, dans la description des génotypes, les données de la génomique et, en aval, de toute la machinerie métabolique contrôlée par l'expression des gènes, à savoir les informations issues de la protéomique et de la métabolomique. En fin de compte, ceci devrait permettre une description beaucoup plus fine des effets génétiques et environnementaux sur les voies métaboliques d'intérêt vis-à-vis de la partition des nutriments. En ce qui concerne l'applicabilité de cette approche, il y aura des obstacles méthodologiques importants qui devront être levés pour que l'information issue des approches en « -omiques » devienne abordable et disponible en routine. Aujourd'hui seule l'information génomique est en train de devenir accessible. Parce que le génome est globalement commun dans tous les tissus et à toutes les étapes de la vie (au sein d'un animal donné), cette information est plus facilement accessible que celle des autres «-omiques ». Bien que des études portant sur l'ensemble du génome aient souvent rencontré des difficultés pour expliquer la majorité des variations de l'ensemble du phénotype de l'animal, l'approche génomique offre la possibilité d'améliorer notablement notre capacité à décrire les effets génétiques sur la partition des nutriments (Pryce et al 2010). Au-delà, le défi majeur réside dans l'intégration verticale, «bottom-up », à partir de ce niveau « atomisé » de description de l'animal pour atteindre des descriptions phénotypiques globales ayant un sens biologique et pratique. Le danger est que toutes les informations «-omiques » disponibles constituent un ensemble informationnel trop complexe pour être intelligible et manipulable. Il est vraisemblable que nous aurons à mettre en œuvre une approche systémique, et à utiliser la notion d'émergence comme une façon d'appréhender la complexité. Sur cette base il sera possible de construire des modèles représentants différents niveaux d'organisation, une telle démarche devant être conduite d'une manière globalement structurée. Cela devrait permettre à différents modèles représentant différents niveaux d'être articulés de manière utile et logique (Lavelle et al 2008).

\section{Références}

Baldwin R.L., 1995. Modeling ruminant digestion and metabolism. Chapman \& Hall, London, UK, 592p.

Baldwin R.L., France J., Beever D.E., Gill M., Thornley J.H.M., 1987. Metabolism of the lactating cow. III. Properties of mechanistic models suitable for evaluation of energetic relationships and factors involved in the partition of nutrients. J. Dairy Res., 54, 133-145.

Banos G., Coffey M.P., 2010. Genetic association between body energy measured throughout lactation and fertility in dairy cattle. Animal, 4, 189-199.

Banos G., Brotherstone S., Coffey M.P., 2005. Genetic profiles of total body energy content of Holstein cows in the first three lactations. J. Dairy Sci., 88, 2616-2623.

Bareille N., Faverdin P., Hay M., 1997. Modification of feed intake response to a beta(2)agonist by bovine somatotropin in lactating or dry dairy cows. J. Dairy Sci., 80, 52-66.

Baudracco J., Lopez-Villalobos N., Holmes C.W., Comeron E.A., Macdonald K.A., Barry T.A., Friggens N.C., 2012. e-Cow: an animal model that predicts herbage intake, milk yield and live weight change in dairy cows grazing temperate pastures, with and without supplementary feeding. Animal, 6 980-993.
Bauman D.E., Currie W.B., 1980. Partitioning of nutrients during pregnancy and lactation: a review of mechanisms involving homeostasis and homeorhesis. J. Dairy Sci., 63, 1514-1529.

Beerda B., Ouweltjes W., Sebek L.B.J., Windig J.J., Veerkamp R.F., 2007. Effects of genotype by environment interactions on milk yield, energy balance, and protein balance. J. Dairy Sci., 90, 219-228.

Bernard C., 1865. Introduction à l'étude de la médecine expérimentale. Baillère, Paris, France, 205 p.

Berry D.P., Buckley F., Dillon P., Evans R.D., Rath M., Veerkamp R.F., 2002. Genetic parameters for level and change of body condition score and body weight in dairy cows. J. Dairy Sci., 85, 2030-2039.

Berry D.P., Veerkamp R.F., Dillon P., 2006 Phenotypic profiles for body weight, body condition score, energy intake, and energy balance across different parities and concentrate feeding levels. Livest. Sci., 104, 1-12.

Bjerre-Harpoth V., Friggens N.C., Thorup V.M., Larsen T., Ingvartsen K.L., Moyes K.M., 2012. Decreased nutrient density to increase physiological imbalance for dairy cows at different stages of lactation: 1. milk yield and component responses. J. Dairy Sci., 95, 2362-2380.
Broster W.H., Broster V.J., 1998. Body score of dairy cows. J. Dairy Res., 65, 155-173.

Brun-Lafleur L., 2011. Modélisation du fonctionnement d'un troupeau laitier pour anticiper les conséquences de son pilotage. $\mathrm{PhD}$ thesis, Agrocampus-Ouest, Rennes, France. 280 p.

Brun-Lafleur L., Delaby L., Husson F., Faverdin P., 2010. Predicting energy x protein interaction on milk yield and milk composition in dairy cows. J. Dairy Sci., 93, 4128-4143.

Brun-Lafleur L., Cutullic E., Faverdin P. Delaby L., Disenhaus C., 2013. An individual reproduction model sensitive to milk yield and body condition in Holstein dairy cows. Animal, FirstView, 1-12.

Bryant J.R., Lopez-Villalobos N., Holmes C., Pryce J.E., 2005. Simulation modelling of dairy cattle performance based on knowledge of genotype, environment and genotype by environment interactions: current status. Agric. Syst., 86, 121-143.

Bryant J.R., Lopez-Villalobos N., Pryce J.E., Holmes C.W., Johnson D.L., 2006. Reaction norms used to quantify the responses of New Zealand dairy cattle of mixed breeds to nutritional environment. New Zealand J. Agric. Res., 49, 371-381. 
Bryant J.R., Lopez-Villalobos N., Holmes C.W., Pryce J.E., Pitman G.D., Davis S.R., 2007. The effect of level of feeding, genetic merit, body condition score and age on biological parameters of a mammary gland model. Animal, 1, 175-183.

Bryant J.R., Lopez-Villalobos N., Holmes C., Pryce J.E., Rossi J., Macdonald K.A., 2008. Development and evaluation of a pastoral simulation model that predicts dairy cattle performance based on animal genotype and environmental sensitivity information. Agric. Syst., 97, 13-25.

Cannon W.B., 1929. Organization for physiological homeostasis. Physiol. Rev., 9, 399-431.

Chilliard Y., Ferlay A., Faulconnier Y., Bonnet M., Rouel J., Bocquier F., 2000. Adipose tissue metabolism and its role in adaptations to undernutrition in ruminants. Proc. Nutr. Soc., 59, 127-134.

Coffey M.P., Emmans G.C., Brotherstone S., 2001. Genetic evaluation of dairy bulls for energy balance traits using random regression. Anim. Sci., 73, 29-40.

Coffey M.P., Simm G., Hill W.G., Brotherstone S., 2003. Genetic evaluations of dairy bulls for daughter energy balance profiles using linear type scores and body condition score analysed using random regression. J. Dairy Sci., 86, 2205-2212.

Collier R.J., Baumgard L.H., Lock A.L., Bauman D.E., 2005. Physiological limitations, nutrient partitioning. In: Yields of farmed species. Constraints and opportunities in the $21^{\text {st }}$ century. Sylvester-Bradley R., Wiseman J. (Eds). Nottingham University Press, Nottingham, U.K., 351-377.

Cutullic E., Delaby L., Michel G., Disenhaus C., 2009. Consequence on reproduction of two feeding levels with opposite effects on milk yield and body condition loss in Holstein and Normande cows. J. Dairy Sci., 92 (E-suppl 1), 355.

Danfær A., 1990. A dynamic model of nutrient digestion and metabolism in lactating dairy cows, Foulum, Denmark, 671, 511.

Delaby L., Faverdin P., Michel G., Disenhaus C., Peyraud J.L., 2009. Effect of different feeding strategies on lactation performance of Holstein and Normande dairy cows. Animal, 3, 891-905.

Dijkstra J., France J., Dhanoa M.S., Maas J.A., Hanigan M.D., Rook A.J., Beever D.E., 1997. A model to describe growth patterns of the mammary gland during pregnancy and lactation. J. Dairy Sci., 80, 2340-2354.

Dillon P., Berry D.P., Evans R.D., Buckley F., Horan B., 2006. Consequences of genetic selection for increased milk production in European seasonal pasture based systems of milk production. Livest. Sci., 99, 141-158.

Doeschl-Wilson A.B., Knap P.W., Kinghorn B.P., Van der Steen H.A.M., 2007. Using mechanistic animal growth models to estimate genetic parameters of biological traits. Animal, 1, 489-499.

Drackley J.K., Overton T.R., Douglas G.N., 2001. Adaptations of glucose and long-chain fatty acid metabolism in liver of dairy cows during the periparturient period. J. Dairy Sci., 84 (E Suppl.), E100-E112.

Emmans G.C., 1997. A method to predict the food intake of domestic animals from birth to maturity as a function of time. J. Theor. Biol., 186, 189-199.
Emmans G.C., Kyriazakis I., 2001. Consequences of genetic change in farm animals on food intake and feeding behaviour. Proc. Nutr. Soc., 60, 115-125.

Faverdin P., 1985. Régulation de l'ingestion des vaches laitières en début de lactation : variations au cours du nychtémère de l'activité alimentaire, des métabolites sanguins et de l'insulinémie et étude du rôle de l'insuline. Thèse de Doctorat de l'INAPG. 130p.

Faverdin P., Bareille N., 1999. Lipostatic regulation of feed intake in ruminants, in: $\mathrm{D}$. van der Heide, E.A. Huisman, E. Kanis, J.W.M. Osse (Eds.), Regulation of feed intake. CABI Publishing, 89-102.

Faverdin P., Dulphy J.P., Coulon J.B., Verite R., Garel J.P., Rouel J., Marquis B., 1991. Substitution of roughage by concentrates for dairy cows. Livest. Prod. Sci., 27, 137-156.

Faverdin P., Delaby L., Delagarde R., 2007. L'ingestion d'aliments par les vaches laitières et sa prévision au cours de la lactation. In Alimentation des ruminants. Agabriel J. (Ed). Dossier, INRA Prod. Anim., 20, 151-162.

Friggens N.C., 2003. Body lipid reserves and the reproductive cycle: towards a better understanding. Livest. Prod. Sci., 83, 219-226.

Friggens N.C., Badsberg J.H., 2007. The effect of breed and parity on lactation curves of body condition estimated using a non-linear function. Animal, 1, 565-574.

Friggens N.C., Newbold J.R., 2007. Towards a biological basis for predicting nutrient partitioning: the dairy cow as an example. Animal, 1, 87-97.

Friggens N.C., Emmans G.C., Kyriazakis I., Oldham J.D., Lewis M., 1998. Feed intake relative to stage of lactation for dairy cows consuming total mixed diets with a high or low ratio of concentrate to forage. J. Dairy Sci., 81, 2228-2239.

Friggens N.C., Emmans G.C., Veerkamp R.F., 1999. On the use of simple ratios between lactation curve coefficients to describe parity effects on milk production. Livest. Prod. Sci., $62,1-13$.

Friggens N.C., Ingvartsen K.L., Emmans G.C., 2004. Prediction of body lipid change in pregnancy and lactation. J. Dairy Sci., 87, 9881000 .

Friggens N.C., Berg P., Theilgaard P., Korsgaard I.R., Ingvartsen K.L., Lıvendahl P.L., Jensen J., 2007. Breed and parity effects on energy balance profiles through lactation: evidence for genetically driven body reserve change. J. Dairy Sci., 90, 5291-5305.

Friggens N.C., Disenhaus C., Petit H.V., 2010. Nutritional sub-fertility in the dairy cow: towards improved reproductive management through a better biological understanding. Animal, 4, 1197-1213.

Friggens N.C., Brun-Lafleur L., Faverdin P., Sauvant D., Martin O., 2013. Advances in predicting nutrient partitioning in the dairy cow: recognizing the central role of genotype and its expression through time. Animal, 7:s1, 89-101.

Garnsworthy P.C., Topps J.H., 1982. The effect of body condition of dairy cows at calving on their food intake and performance when given complete diets. Anim. Prod., 35, 113119.

Goelzer A., Brikci F.B., Martin-Verstraete I., Noirot P., Bessieres P., Aymerich S.,
Fromion V., 2008. Reconstruction and analysis of the genetic and metabolic regulatory networks of the central metabolism of Bacillus subtilis. BMC Systems Biology, 2, 20 DOI 10.1186/1752-0509-2-20.

Goelzer A., Fromion V., 2011. Bacterial growth rate reflects a bottleneck in resource allocation. Bioch. Biophysica Acta-General Subjects, 1810, 978-988.

Gong J.G., Lee W.J., Garnsworthy P.C., Webb R., 2002. Effect of dietary-induced increases in circulating insulin concentrations during the early postpartum period on reproductive function in dairy cows. Reproduction, 123, 419-427.

Grala T.M., Lucy M.C., Phyn C.V.C., Sheahan A.J., Lee J.M., Roche J.R., 2011. Somatotropic axis and concentrate supplementation in grazing dairy cows of genetically diverse origin. J. Dairy Sci., 94 303-315.

Hammond J., 1944. Physiological factors affecting birth weight. Proc. Nutr. Soc., 2, 8-12.

Hanigan M.D., Bateman H.G., Fadel J.G., McNamara J.P., 2006. Metabolic models of ruminant metabolism: Recent improvements and current status. J. Dairy Sci., 89 (E. Suppl), E52-E64.

Hanigan M.D., Rius A.G., Kolver E.S., Palliser C.C., 2007. A redefinition of the representation of mammary cells and enzyme activities in a lactating dairy cow model. J. Dairy Sci., 90, 3816-3830.

Hanigan M.D., Palliser C.C., Gregorini P., 2009. Altering the representation of hormones and adding consideration of gestational metabolism in a metabolic cow model reduced prediction errors. J. Dairy Sci., 92, 5043-5056.

Hoggard N., Hunter L., Trayhurn P., Williams L.M., Mercer J.G., 1998. Leptin and reproduction. Proc. Nutr. Soc., 57, 421-427.

Horan B., Dillon P., Berry D.P., O'Connor P., Rath M., 2005. The effect of strain of Holstein-Friesian, feeding system and parity on lactation curves characteristics of springcalving dairy cows. Livest. Prod. Sci., 95, 231241.

Horan B., Faverdin P., Delaby L., Rath M., Dillon P., 2006. The effect of strain of Holstein-Friesian dairy cow and pasture-based system on grass intake and milk production. Anim. Sci., 82, 435-444.

Ingvartsen K.L., 2006. Feeding- and management- related diseases in the transition cow Physiological adaptations around calving and strategies to reduce feeding-related diseases. Anim. Feed Sci. Technol., 126, 175-213.

Kaske M., Groth A., 1997. Changes in factors affecting the rate of digesta passage during pregnancy and lactation in sheep fed on hay. Reprod. Nutr. Dev., 37, 573-588.

Kebreab E., Dijkstra J., Bannink A., France J., 2009. Recent advances in modeling nutrient utilization in ruminants. J. Anim. Sci., 87(E Suppl.), E111-E122.

Kennedy G.C., 1953. The role of depot fat in the hypothalamic control of food intake in rats. Proc. Royal Soc. London, Series B, Biol. Sci., 140, 578-592.

Kennedy G.C., 1967. Ontogeny of mechanisms controlling food and water intake. In: Alimentary Canal Volume 1. Control of Food and Water Intake. Code C.F. (Ed). Amer. Physiol. Soc., Washington, D.C., USA, 337-352. 
Koenen E.P.C., Veerkamp R.F., Dobbelaar P., De Jong G., 2001. Genetic analysis of body condition score of lactating Dutch Holstein and Red-and-White heifers. J. Dairy Sci., 84, 1265 1270.

Lavelle C., Berry H., Beslon G., Ginelli F., Giavitto J.L., Kapoula Z., Le Bivic A., Peyrieras N., Radulescu O., Six A., ThomasVaslin V., Bourgine P., 2008. From molecules to organisms: towards multiscale integrated models of biological systems. Theor.1 Biol. Insights, 1, 13-22.

Leclerc H., Duclos D., Barbat A., Druet T., Ducrocq V., 2008. Environmental effects on lactation curves included in a test-day model genetic evaluation. Animal, 2, 344-353.

Lemosquet S., Abdou Arbi O., Siegel A., Guinard-Flament J., Van Milgen J., Bourdon J., 2010. A generic stoichiometric model to analyse the metabolic flexibility of the mammary gland in lactating dairy cows. Sauvant D., Van Milgen J., Faverdin P., Friggens N.C. (Eds). Wageningen Academic Publishers, The Netherlands, 279-288.

Loor J.J., 2010. Genomics of metabolic adaptations in the peripartal cow. Animal, 4, 1110-1139.

Loor J.J., Dann H.M., Janovick Guretzky N.A., Everts R.E., Oliveira R., Green C.A., Litherland N.B., Rodriguez-Zas S.L., Lewin H.A., Drackley J.K., 2006. Plane of nutrition pre-partum alters hepatic gene expression and function in dairy cows as assessed by longitudinal transcript and metabolic profiling. Physiol. Genomics, 27, 29-41.

Lucy M.C., 2003. Mechanisms linking nutrition and reproduction in postpartum cows. Reproduction, 61, 415-427.

Lucy M.C., Verkerk G.A., Whyte B.E., Macdonald K.A., Burton L., Cursons R.T., Roche J.R., Holmes C.W., 2009. Somatotropic axis components and nutrient partitioning in genetically diverse dairy cows managed under different feed allowances in a pasture system. J. Dairy Sci., 92, 526-539.

Martin O., Sauvant D., 2007. Dynamic model of the lactating dairy cow metabolism. Animal, 1, 1143-1166.

Martin O., Sauvant D., 2010a. A teleonomic model describing performance (body, milk and intake) during growth and over repeated reproductive cycles throughout the lifespan of dairy cattle. 1. Trajectories of life function priorities and genetic scaling. Animal, 4, 2030-2047.

Martin O., Sauvant D., 2010b. A teleonomic model describing performance (body, milk and intake) during growth and over repeated reproductive cycles throughout the lifespan of dairy cattle. 2. Voluntary intake and energy partitioning. Animal, 4, 2048-2056.

Maxin G., 2011. Développement d'un modèle de prédiction de la matière grasse du lait basé sur les flux de nutriments chez la vache laitière. Thèse de doctorat, Agrocampus-Ouest, Rennes, France. 193p.

McNamara J.P., 2004. Research, improvement and application of mechanistic, biochemical, dynamic models of metabolism in lactating dairy cows. Anim. Feed Sci. Technol., 112, 155-176.

McNamara J.P., Baldwin R.L., 2000 Estimation of parameters describing lipid metabolism in lactation: Challenge of existing knowledge described in a model of metabolism. J. Dairy Sci., 83, 128-143.
Monod J., 1970. Le hasard et la necessité Essai sur la philosophie naturelle de la biologie moderne. Ed. Le Seuil, Paris, France, 221p.

Neal H.D.S.C., Thornley J.H.M., 1983. The lactation curve in cattle: a mathematical model of the mammary gland. J. Agric. Sci. Cambridge, 101, 389-400.

Oldham J.D., Emmans G.C., 1989. Prediction of responses to required nutrients in dairy cows. J. Dairy Sci., 72, 3212-3229.

Ostergaard S., Sorensen J.T., Kristensen A.R., 2000. A stochastic model simulating the feeding-health-production complex in a dairy herd. J. Dairy Sci., 83, 721-733.

Petruzzi H., Danfær A., 2004. A dynamic model of feed intake regulation in dairy cows. Model description. J. Anim. Feed Sci., 13, 123.

Pittendrigh C.S., 1958. Adaptation, natural selection, and behavior. In: Behavior and Evolution. Roe A., Gaylord Simpson G. (Eds). Yale University Press, New Haven, USA, 390416.

Pollott G.E., 2000. A biological approach to lactation curve analysis for milk yield. J. Dairy Sci., 83, 2448-2458.

Pond C.M., 1984. Physiological and ecological importance of energy storage in the evolution of lactation: evidence for a common pattern of anatomical organization of adipose tissue in mammals. In: Physiological strategies in lactation. Peaker M., Vernon R.G., Knight C.H (Eds). Zool. Soc. London, UK, 1-29.

Pryce J.E., Bolormaa S., Chamberlain A.J., Bowman P.J., Savin K., Goddard M.E., Hayes B.J., 2010. A validated genome-wide association study in 2 dairy cattle breeds for milk production and fertility traits using variable length haplotypes. J. Dairy Sci., 93, 3331-3345.

Réale D., Festa-Bianchet M., 2000. Massdependent reproductive strategies in wild bighorn sheep: a quantitative genetic approach. J. Evol. Biol., 13, 679-688.

Reznick D., Nunney L., Tessier A., 2000. Big houses, big cars, superfleas and the costs of reproduction. Trends Ecol. Evol., 15, 421-425.

Rhoads R.P., Sampson J.D., Lucy M.C., Spain J.N., Spiers D.E., Tempelman R.J. Coussens P.M., 2005. Hepatic gene expression profiling during adaptation to a period of chronic heat stress in lactating dairy cows. FASEB J. 19, A1673-A1673.

Roche J.R., Berry D.P., Kolver E.S., 2006 Holstein-Friesian strain and feed effects on milk production, body weight, and body condition score profiles in grazing dairy cows. J. Dairy Sci., 89, 3532-3543.

Roche J.R., Friggens N.C., Kay J.K., Fishe M.W., Stafford K.J., Berry D.P., 2009. Body condition score and its association with dairy cow productivity, health, and welfare: A review. J. Dairy Sci., 92, 5769-5801.

Sauvant D., 1992. La modélisation systémique en nutrition. Reprod. Nutr. Dev., 32 $217-230$

Sauvant D., 1994. Modelling homeostatic and homeorhetic regulations in lactating animals. Livest. Prod. Sci., 39, 105-113.

Sauvant D., Van Milgen J., Faverdin P., Friggens N.C., 2010. Modelling nutrient digestion and utilisation in farm animals. Proc. $7^{\text {th }}$ Int Workshop. Wageningen Academic Publishers, the Netherlands, 427p.
Schmidely P., Duvaux-Ponter C., LaporteBroux B., Tessier J., Friggens N.C., 2011. Characterizing individual differences in performance responses to a nutritional challenge. Book of abstracts, 62 ${ }^{\text {nd }}$ Ann. Meet. Europ. Assoc. Anim. Prod., 303pp.

Shalloo L., Kennedy J., Wallace M., Rath M., Dillon P., 2004. The economic impact of cow genetic potential for milk production and concentrate supplementation level on the profitability of pasture based systems under differen EU milk quota scenarios. J. Agric. Sci., 142, 357-369

Sumner-Thomson J.M., Vierck J.L., McNamara J.P., 2011. Differential expression of genes in adipose tissue of first-lactation dairy cattle. J. Dairy Sci., 94, 361-369.

Taylor S.C.S., 1980. Genetic size-scaling rules in animal growth. Anim. Prod., 30, 161165.

Theilgaard P., Sanchez J., Pascual J.J., Berg P., Friggens N.C., Baselga M., 2007. Late reproductive senescence in a rabbit line hyper selected for reproductive longevity, and its association with body reserves. Genet. Selec. Evol., 39, 207-223.

Tolkamp B.J., Ketelaars J.J.M.H., 1992 Toward a new theory of feed intake regulation in ruminants. 2. Costs and benefits of feed consumption: an optimization approach. Livest. Prod. Sci., 30, 297-317.

Tylutki T.P., Fox D.G., Durbal V.M., Tedeschi L.O., Russell J.B., Van Amburgh M.E., Overton T.R., Chase L.E., Pell A.N. 2008. Cornell Net Carbohydrate and Protein System: a model for precision feeding of dairy cattle. Anim. Feed Sci. Technol., 143, 174-202.

van der Waaij E.H., 2004. A resource allocation model describing consequences of artificial selection under metabolic stress. J. Anim. Sci., 82, 973-981.

van Knegsel A.T.M., van den Branda H., Dijkstra J., Tamminga S., Kemp B., 2005. Effect of dietary energy source on energy balance, production, metabolic disorders and reproduction in lactating dairy cattle. Reprod. Nutr. Dev., 45, 665-688.

Veerkamp R.F., Goddard M.E., 1998. Covariance functions across herd production levels for test day records on milk fat and protein yields. J. Dairy Sci., 81, 1690-1701.

Vetharaniam I., Davis S.R., Soboleva T.K. Shorten P.R., Wake G.C., 2003a. Modeling the interaction of milking frequency and nutrition on mammary gland growth and lactation. J. Dairy Sci., 86, 1987-1996.

Vetharaniam I., Davis S.R., Upsdell M., Kolver E.S., Pleasants A.B., 2003b. Modeling the effect of energy status on mammary gland growth and lactation. J. Dairy Sci., 86, 31483156 .

Waddington C.H., 1957. The strategy of the genes. A discussion of some aspects of theoretical biology. George Allen \& Unwin Ltd, London.

Yan T., Mayne C.S., Keady T.W.J., Agnew R.E., 2006. Effects of dairy cow genotype with two planes of nutriton on energy partitioning between milk and body tissue. J. Dairy Sci., 89 , 1031-1042.

Yearsley J., Tolkamp B.J., Illius A.W. 2001. Theoretical developments in the study and prediction of food intake. Proc. Nutr. Soc. $60,145-156$ 


\section{Résumé}

La compréhension de la partition des nutriments est essentielle pour appréhender le fonctionnement biologique des animaux d'élevage et pas seulement pour prévoir les sorties productives. La partition des nutriments entre la fonction lactation et d'autres fonctions telles que la capacité immunitaire et la reproduction est essentielle vis-à-vis de la capacité des élevages à s'adapter, au même titre que l'efficacité de la partition des nutriments. La sélection des vaches sur la production de lait a accru la sollicitation des réserves corporelles tout en réduisant la robustesse des vaches laitières. En conséquence, il devient de plus en plus nécessaire d'intégrer dans les modèles nutritionnels des forces motrices innées qui modifient la partition des nutriments en fonction de l'état physiologique et qui semblent déterminer des trajectoires programmées. Cette synthèse présente à partir de travaux récents, comment les modèles nutritionnels de la lactation peuvent mieux intégrer ces trajectoires et la capacité homéostatique de l'animal pour faire face aux changements d'environnement. Ces développements ont considérablement étendu la portée générale des modèles de partition des nutriments afin de mieux prendre en compte le type d'animal et son état physiologique. Cependant, ces modèles restent très largement axés sur la prévision de la partition entre les sorties de production et les réserves corporelles et sont encore, pour la plupart, des modèles de recherche, bien que des progrès substantiels aient été accomplis pour en faire des outils d'aide à la décision en élevage. Relier la prévision de la partition des éléments nutritifs à ses conséquences sur la santé, la reproduction et la longévité est un défi qui ne fait que commencer à être pris en compte. $C$ 'est une perspective importante pour les recherches futures sur la partition des nutriments.

\section{Abstract \\ Nutrient partitioning between physiological functions in the dairy cow depends on the genotype and its expression through time}

It has become increasingly clear that understanding nutrient partitioning is central to a much broader range of issues than just being able to predict productive outputs. The extent to which nutrients are partitioned to other functions such as health and reproduction is clearly important, as are the efficiency consequences of nutrient partitioning. Further, there is mounting evidence that reliance on body reserves is increased and that robustness of dairy cows is reduced by selection for increased milk production. There is a need to incorporate in nutritional models the innate driving forces that alter nutrient partitioning according to physiological state i.e., the role of both the cow's genotype (genetic make-up), and the expression of this genotype through time on nutrient partitioning. This paper summarizes work carried out to extend nutritional models to incorporate these trajectories, and how they affect the homeostatic capacity of the animal. There are now models that can predict the partition of nutrients throughout lactation for cows of differing milk production potentials. However, genotype and stage of lactation effects on homeostatic capacity has not yet been explicitly included in metabolic models that predict nutrient partition, although recent results suggest that this is achievable. These developments considerably extend the generality of nutrient partitioning models with respect to the type of animal and its physiological state. However, these models remain very largely focussed on predicting partition between productive outputs and body reserves and, for the most part, remain research models although substantial progress has been made toward developing models that can be applied in the field. The challenge of linking prediction of nutrient partitioning to its consequences on health, reproduction, and longevity, although widely recognised, is only now beginning to be addressed. This is an important perspective for future work on nutrient partitioning.

FRIGGENS N.C., MARTIN O., BRUN-LAFLEUR L., SAUVANT D., FAVERDIN P., 2013. La partition des nutriments entre fonctions physiologiques chez les vaches laitières dépend du génotype et de son expression dans le temps. In : Numéro spécial, La vache et le lait. Faverdin P., Leroux C., Baumont R. (Eds). INRA Prod. Anim., 26, 2, 101-116. 